\title{
Symmetry Breaking in Coupled SYK or Tensor Models
}

\author{
Jaewon Kim, ${ }^{1}$ Igor R. Klebanov, ${ }^{2,3}$ Grigory Tarnopolsky, ${ }^{4}$ and Wenli Zhao ${ }^{2}$ \\ ${ }^{1}$ Department of Physics, University of California, Berkeley, California 94720, USA \\ ${ }^{2}$ Department of Physics, Princeton University, Princeton, New Jersey 08544, USA \\ ${ }^{3}$ Princeton Center for Theoretical Science, Princeton University, Princeton, New Jersey 08544, USA \\ ${ }^{4}$ Department of Physics, Harvard University, Cambridge, Massachusetts 02138, USA
}

(Received 22 March 2019; published 31 May 2019)

\begin{abstract}
We study a large- $N$ tensor model with $O(N)^{3}$ symmetry containing two flavors of Majorana fermions, $\psi_{1}^{a b c}$ and $\psi_{2}^{a b c}$. We also study its random counterpart consisting of two coupled Sachdev-Ye-Kitaev (SYK) models, each containing $N_{\mathrm{SYK}}$ Majorana fermions. In these models, we assume tetrahedral quartic Hamiltonians which depend on a real coupling parameter $\alpha$. We find a duality relation between two Hamiltonians with different values of $\alpha$, which allows us to restrict the model to the range of $-1 \leq \alpha \leq 1 / 3$. The scaling dimension of the fermion number operator $Q=i \psi_{1}^{a b c} \psi_{2}^{a b c}$ is complex and of the form $1 / 2+$ if $(\alpha)$ in the range $-1 \leq \alpha<0$, indicating an instability of the conformal phase. Using Schwinger-Dyson equations to solve for the Green functions, we show that in the true low-temperature phase this operator acquires an expectation value, which demonstrates the breaking of an antiunitary particle-hole symmetry and other discrete symmetries. We also calculate spectra of the coupled SYK models for values of $N_{\text {SYK }}$ where exact diagonalizations are possible. For negative $\alpha$, we find a gap separating the two lowest energy states from the rest of the spectrum, leading to an exponential decay of the zero-temperature correlation functions. For $N_{\mathrm{SYK}}$ divisible by 4 , the two lowest states have a small splitting. They become degenerate in the large- $N_{\mathrm{SYK}}$ limit, as expected from the spontaneous breaking of a $\mathbb{Z}_{2}$ symmetry.

DOI: 10.1103/PhysRevX.9.021043

Subject Areas: Condensed Matter Physics, Particles and Fields, String Theory
\end{abstract}

\section{INTRODUCTION AND SUMMARY}

During the past several years, there has been a flurry of activity on fermionic quantum-mechanical models which are exactly solvable in the large- $N$ limit because they are dominated by the so-called melonic Feynman diagrams. Work in this direction began with the Sachdev-Ye-Kitaev (SYK) models [1-4], which have random couplings. More recently, the tensor quantum-mechanical models $[5,6]$, which have continuous symmetry groups and no randomness, were constructed following the body of research on melonic large- $N$ tensor models in $d=0$ [7-13] (for reviews, see Refs. [14-17]). Both the random and nonrandom quantum-mechanical models are solvable via the melonic Schwinger-Dyson equations [4,18-21], which indicate the existence of the nearly conformal phase which saturates the chaos bound. They shed new light on the dynamics of two-dimensional black holes [22-25].

Published by the American Physical Society under the terms of the Creative Commons Attribution 4.0 International license. Further distribution of this work must maintain attribution to the author(s) and the published article's title, journal citation, and DOI.
These models may also have applications to a range of problems in condensed matter physics, including the strange metals [3,26-32]. With such applications in mind, it is interesting to study various dynamical phenomena in the SYK and tensor models. For example, phase transitions in such models have been studied in Refs. [33-35]. In this paper, we identify a simple setting where spontaneous symmetry breaking can occur: two SYK or tensor models coupled via a quartic interaction. We take this interaction to be purely melonic (i.e., tetrahedral in the tensor model case) so that the symmetry breaking can be deduced from the large- $N$ Schwinger-Dyson equations.

In the random case, we study two coupled SYK models with the Hamiltonian

$H=\frac{1}{4 !} J_{i j k l}\left(\chi_{1}^{i} \chi_{1}^{j} \chi_{1}^{k} \chi_{1}^{l}+\chi_{2}^{i} \chi_{2}^{j} \chi_{2}^{k} \chi_{2}^{l}+6 \alpha \chi_{1}^{i} \chi_{1}^{j} \chi_{2}^{k} \chi_{2}^{l}\right)$,

where, as usual, all repeated indices are summed over. The Majorana fermions are $\chi_{1}^{i}$ and $\chi_{2}^{i}$ with $i=1, \ldots, N_{\mathrm{SYK}}$, and $J_{i j k l}$ is a fully antisymmetric real tensor with a Gaussian distribution [36]. We show that the real parameter $\alpha$ may be restricted to the range $-1 \leq \alpha \leq 1 / 3$ by a duality symmetry. This quartic Hamiltonian, which couples $2 N_{\text {SYK }}$ Majorana fermions, is invariant under an antiunitary 
particle-hole symmetry [37-42] generated by $\mathcal{P}$; see Eq. (3.11). However, we show that for $-1 \leq \alpha<0$ this $\mathbb{Z}_{2}$ symmetry is spontaneously broken when $N_{\mathrm{SYK}}$ is divisible by 4 and taken to infinity [43]. In this limit, the fermion number operator $Q=i \chi_{1}^{j} \chi_{2}^{j}$ acquires an expectation value. Such an expectation value leads to a gapped phase in two coupled SYK models similar to that found by Maldacena and Qi [44] (for further results, see Refs. [45]); however, instead of the quartic they assumed a quadratic coupling term $\mu Q$ which breaks the $\mathbb{Z}_{2}$ symmetry explicitly. This gapped phase was argued to be dual to a traversable wormhole in two-dimensional gravity [46,47], and our model (1.1) may have a similar interpretation for $-1 \leq \alpha<0$.

As we show in Sec. II D, a sign of instability of the conformal phase for $-1 \leq \alpha<0$ is the presence of a complex scaling dimensions of the form $1 / 2+i f(\alpha)$. The appearance of complex dimensions with real part equal to $d / 2$ for some single-trace operators is a common phenomenon in large- $N$ models [48-52]. Via the AdS/CFT correspondence [53-55], such operators are related to scalar fields which violate the Breitenlohner-Freedman stability bound [56]. The fact that $\alpha=0$ is the lower edge of the conformal window is related to the appearance of the marginal double-trace operator $Q^{2}$ there. For $0<\alpha \leq 1 / 3$, there are actually two fixed points connected by the flow of the coefficient of $Q^{2}$, but at $\alpha=0$ they merge and annihilate, as explained, e.g., in Refs. [57,58].

The complex scaling dimensions have been observed in bosonic tensor models $[59,60]$ as well as in a complex fermionic model introduced in Ref. [6] following the work in Ref. [61]. This fermionic model is often called "bipartite" because of the two types of interaction vertices (black and white) arranged in an alternating fashion, since the propagator must connect different vertices. The bipartite model was further studied in Ref. [17] and shown to possess a complex scaling dimension of the operator $\bar{\psi}^{a b c} \psi^{a b c}$. Here we generalize this tensor model to one with a continuous parameter $\alpha$ in such a way that the bipartite model corresponds to $\alpha=-1$. This $O(N)^{3}$ symmetric model for Majorana fermions $\psi_{1}^{a b c}$ and $\psi_{2}^{a b c}$, with $a, b, c=1, \ldots, N$, has Hamiltonian

$$
\begin{aligned}
H= & \frac{g}{4}\left(\psi_{1}^{a_{1} b_{1} c_{1}} \psi_{1}^{a_{1} b_{2} c_{2}} \psi_{1}^{a_{2} b_{1} c_{2}} \psi_{1}^{a_{2} b_{2} c_{1}}+\psi_{2}^{a_{1} b_{1} c_{1}} \psi_{2}^{a_{1} b_{2} c_{2}} \psi_{2}^{a_{2} b_{1} c_{2}} \psi_{2}^{a_{2} b_{2} c_{1}}\right) \\
& +\frac{g \alpha}{2}\left(\psi_{1}^{a_{1} b_{1} c_{1}} \psi_{1}^{a_{1} b_{2} c_{2}} \psi_{2}^{a_{2} b_{1} c_{2}} \psi_{2}^{a_{2} b_{2} c_{1}}+\psi_{1}^{a_{1} b_{1} c_{1}} \psi_{2}^{a_{1} b_{2} c_{2}} \psi_{1}^{a_{2} b_{1} c_{2}} \psi_{2}^{a_{2} b_{2} c_{1}}+\psi_{1}^{a_{1} b_{1} c_{1}} \psi_{2}^{a_{1} b_{2} c_{2}} \psi_{2}^{a_{2} b_{1} c_{2}} \psi_{1}^{a_{2} b_{2} c_{1}}\right) .
\end{aligned}
$$

For $\alpha=0$, this Hamiltonian describes two decoupled copies of the basic Majorana $O(N)^{3}$ model with the tetrahedral interaction [6]. The coupling term proportional to $\alpha$ preserves its discrete symmetries and also has the tetrahedral structure; i.e., every two tensors have only one index contraction, so that the model (1.2) is melonic. It is the tensor counterpart of the coupled SYK model (1.1), and in the large- $N$ limit, it is governed by the same Schwinger-Dyson equations for the two-point and four-point functions [62].

In Sec. II, we derive the Schwinger-Dyson equations and use them to study the scaling dimensions of various $O(N)^{3}$ invariant fermion bilinears. We also exhibit a duality symmetry which allows us to restrict the model to the range $-1 \leq \alpha \leq 1 / 3$. The nearly conformal phase of the theory is stable for $0 \leq \alpha \leq 1 / 3$, but it is unstable for $-1 \leq$ $\alpha<0$ as signaled by the complex scaling dimension of operator $i \psi_{1}^{a b c} \psi_{2}^{a b c}$. The true behavior of the theory with negative $\alpha$ is the spontaneous breaking of the particle-hole $\mathbb{Z}_{2}$ symmetry, as we demonstrate in Sec. III. In Secs. III A and III B, we numerically study the large- $N$ SchwingerDyson equations and exhibit the exponential decay of correlators at low temperature. We also ascertain the existence of second-order phase transitions by numerically computing the free energy. In Sec. III C, we study the numerical spectrum of the coupled SYK model (1.1) via exact diagonalizations at finite $N_{\mathrm{SYK}}$. We observe that for
$-1 \leq \alpha<0$ there is a gap separating the two lowest energy states from the rest of the spectrum. For $N_{\mathrm{SYK}}$ divisible by 4 , there is also a small gap between the two lowest states, consistent with the fact that the ground state must be nondegenerate [37-42], but this gap decreases as $N_{\mathrm{SYK}}$ is increased. In the large- $N_{\text {SYK }}$ limit, the two lowest states become degenerate and give rise to the two inequivalent vacua, which are present due to the spontaneous breaking of the $\mathbb{Z}_{2}$ particle-hole symmetry.

These phenomena imply that the low-temperature entropy is large for $0 \leq \alpha \leq 1 / 3$ but vanishes for $-1 \leq$ $\alpha<0$. It is tempting to suggest that the latter case is dual to a wormhole. This sensitivity to the sign of the interaction coupling two CFTs is like in Ref. [46], where the traversable wormhole appears only for one of the signs [63].

\section{SCHWINGER-DYSON EQUATIONS AND SCALING DIMENSIONS}

In this section, we study the two-flavor tensor model with Hamiltonian (1.2) [64]. It can be compactly written in the form

$$
H=\frac{1}{4 !} J_{I J K L}\left(\psi_{1}^{I} \psi_{1}^{J} \psi_{1}^{K} \psi_{1}^{L}+\psi_{2}^{I} \psi_{2}^{J} \psi_{2}^{K} \psi_{2}^{L}+6 \alpha \psi_{1}^{I} \psi_{1}^{J} \psi_{2}^{K} \psi_{2}^{L}\right),
$$




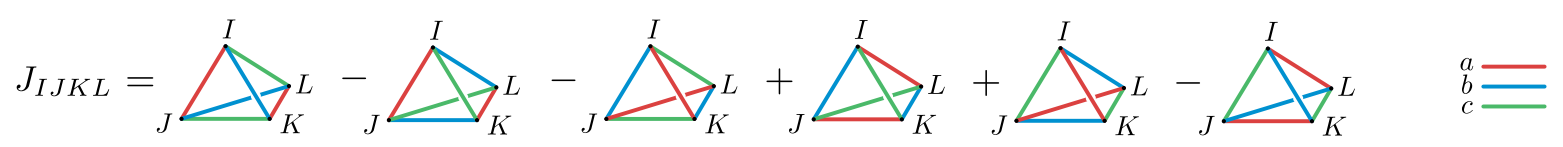

FIG. 1. Pictorial representation of the antisymmetric tensor $J_{I J K L}$.

where the capital letters are a shorthand notation for three tensor indices: $I=a_{1} b_{1} c_{1}, J=a_{2} b_{2} c_{2}$, etc., and the nonrandom tetrahedral tensor coupling consists of six terms

$$
\begin{aligned}
J_{I J K L}= & g \sum_{\sigma \in S_{3}} \operatorname{sgn}(\sigma) \delta_{a_{1} a_{\sigma(2)}} \delta_{b_{1} b_{\sigma(3)}} \delta_{c_{1} c_{\sigma(4)}} \delta_{b_{\sigma(2)} b_{\sigma(4)}} \delta_{c_{\sigma(2)} c_{\sigma(3)}} \\
& \times \delta_{a_{\sigma(3)} a_{\sigma(4)}} .
\end{aligned}
$$

The tensor $J_{I J K L}$ is antisymmetric under permutation of indices $I, J, K, L$ and has a tetrahedron topology as shown in Fig. 1. In the form (2.1), the tensor model Hamiltonian is transparently similar to the SYK one (1.1). In terms of the complex tensors,

$$
\psi^{I}=\frac{1}{\sqrt{2}}\left(\psi_{1}^{I}+i \psi_{2}^{I}\right), \quad \bar{\psi}^{I}=\frac{1}{\sqrt{2}}\left(\psi_{1}^{I}-i \psi_{2}^{I}\right),
$$

the Hamiltonian (2.1) assumes the form

$$
\begin{aligned}
H= & \frac{1}{4 !} J_{I J K L}\left(\frac{1-3 \alpha}{2}\left(\psi^{I} \psi^{J} \psi^{K} \psi^{L}+\bar{\psi}^{I} \bar{\psi}^{J} \bar{\psi}^{K} \bar{\psi}^{L}\right)\right. \\
& \left.+3(1+\alpha) \bar{\psi}^{I} \bar{\psi}^{J} \psi^{K} \psi^{L}\right) .
\end{aligned}
$$

The Hamiltonian (2.1) is invariant under the $O(N)^{3}$ transformation

$$
\psi_{i}^{a b c} \rightarrow A_{a^{\prime}}^{a} B_{b^{\prime}}^{b} C_{c^{\prime}}^{c} \psi_{i}^{a^{\prime} b^{\prime} c^{\prime}},
$$

where $A, B$, and $C$ are orthogonal matrices. In addition, it has a particle-hole $\mathbb{Z}_{2}$ symmetry [66] generated by [37-42],

$$
\mathcal{P}=K \prod_{I}\left(\psi^{I}+\bar{\psi}^{I}\right),
$$

where $K$ is the antiunitary operator which acts by

$$
K i K=-i, \quad K \psi^{I} K=\psi^{I}, \quad K \bar{\psi}^{I} K=\bar{\psi}^{I} .
$$

The fermion number operator

$$
Q=i \psi_{1}^{I} \psi_{2}^{I}=\frac{1}{2}\left[\bar{\psi}^{I}, \psi^{I}\right]
$$

does not in general commute with $H$, but it is conserved mod 4 . The particle-hole symmetry is not anomalous only if the total number of fermions $2 N^{3}$ is a multiple of 8 , i.e., when $N$ is even [37-42]. Even in this case, we argue that in the large- $N$ limit, the symmetry is spontaneously broken for $-1 \leq \alpha<0$ because $Q$ acquires an expectation value.

\section{A. Duality in the Two-Flavor Models}

In this section, we show that the two-flavor models with different values of $\alpha$ can be equivalent. We demonstrate this explicitly in the tensor model case (2.1), but the SYK case (1.1) works analogously. Let us perform the following transformation on the Majorana fermions:

$$
\psi_{1}^{I}=\frac{1}{\sqrt{2}}\left(\tilde{\psi}_{1}^{I}+\tilde{\psi}_{2}^{I}\right), \quad \psi_{2}^{I}=\frac{1}{\sqrt{2}}\left(\tilde{\psi}_{1}^{I}-\tilde{\psi}_{2}^{I}\right) .
$$

It preserves the anticommutation relations and turns the Hamiltonian (2.1) into [67]

$$
\begin{aligned}
H= & \frac{1}{4 !} J_{I J K L} \frac{1+3 \alpha}{2}\left(\tilde{\psi}_{1}^{I} \tilde{\psi}_{1}^{J} \tilde{\psi}_{1}^{K} \tilde{\psi}_{1}^{L}+\tilde{\psi}_{2}^{I} \tilde{\psi}_{2}^{J} \tilde{\psi}_{2}^{K} \tilde{\psi}_{2}^{L}\right. \\
& \left.+\frac{6(1-\alpha)}{1+3 \alpha} \tilde{\psi}_{1}^{I} \tilde{\psi}_{1}^{J} \tilde{\psi}_{2}^{K} \tilde{\psi}_{2}^{L}\right) .
\end{aligned}
$$

Thus, the energy levels are symmetric under the duality transformation

$$
J \rightarrow \frac{1+3 \alpha}{2} J, \quad \alpha \rightarrow \frac{1-\alpha}{1+3 \alpha} .
$$

Defining

$$
\tilde{\alpha}=\frac{1}{2}(1+3 \alpha), \quad \tilde{J}=J \sqrt{|\tilde{\alpha}|,}
$$

we find that the duality transformation

$$
\tilde{\alpha} \rightarrow 1 / \tilde{\alpha}, \quad \tilde{J} \rightarrow \tilde{J}
$$

acts on the rescaled Hamiltonian $\tilde{H}=H / \sqrt{|\tilde{\alpha}|}$ : [68]

$$
\begin{aligned}
\tilde{H}= & \frac{1}{4 !} \tilde{J}_{I J K L}\left[\tilde{\psi}_{1}^{I} \tilde{\psi}_{1}^{J} \tilde{\psi}_{1}^{K} \tilde{\psi}_{1}^{L}+\tilde{\psi}_{2}^{I} \tilde{\psi}_{2}^{J} \tilde{\psi}_{2}^{K} \tilde{\psi}_{2}^{L}\right. \\
& \left.+\left(-2+\frac{4}{\tilde{\alpha}}\right) \tilde{\psi}_{1}^{I} \tilde{\psi}_{1}^{J} \tilde{\psi}_{2}^{K} \tilde{\psi}_{2}^{L}\right] .
\end{aligned}
$$

Therefore, the fundamental domain is $-1 \leq \tilde{\alpha} \leq 1$. Thus, we may restrict $\alpha$ to the domain

$$
-1 \leq \alpha \leq \frac{1}{3} .
$$

The values of $\alpha$ outside of this domain are related to it by the duality. For $\alpha=-1$, the transformation (2.11) maps the theory into itself, but with $H \rightarrow-H$. 


$$
\left\langle\psi_{1}^{I}\left(\tau_{1}\right) \psi_{1}^{I^{\prime}}\left(\tau_{2}\right)\right\rangle_{0}=\delta^{I I^{\prime}} \frac{\tau_{1} \tau_{2}}{\tau_{2}} \tau_{2}
$$

FIG. 2. Bare propagators for the Majorana tensor fields. Each thick black solid or dashed line carries three tensor indices $a, b, c$.

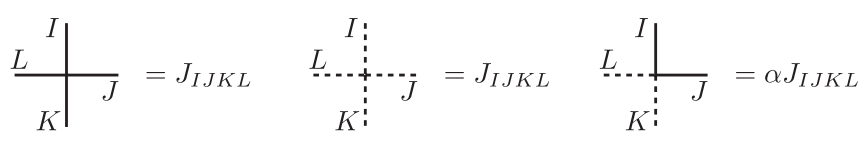

FIG. 3. Interaction vertices.

In fact, the case $\alpha=-1$ corresponds to the complex bipartite model $[17,61]$ :

$H_{\alpha=-1}=2 \frac{1}{4 !} J_{I J K L}\left(\psi^{I} \psi^{J} \psi^{K} \psi^{L}+\bar{\psi}^{I} \bar{\psi}^{J} \bar{\psi}^{K} \bar{\psi}^{L}\right)$

where we introduce the complex tensor $\psi^{I}=(1 / \sqrt{2})$ $\left(\psi_{1}^{I}+i \psi_{2}^{I}\right)$.

The theory with $\alpha=1 / 3$ is mapped into itself by Eq. (2.11). In this case, the Hamiltonian is

$$
H_{\alpha=\frac{1}{3}}=4 \frac{1}{4 !} J_{I J K L} \bar{\psi}^{I} \bar{\psi}^{J} \psi^{K} \psi^{L},
$$

which has $O(N)^{3} \times U(1)$ symmetry. In the three-index notation

$$
\begin{aligned}
H_{\alpha=\frac{1}{3}}= & \frac{g}{3}\left(\bar{\psi}^{a_{1} b_{1} c_{1}} \bar{\psi}^{a_{1} b_{2} c_{2}} \psi^{a_{2} b_{1} c_{2}} \psi^{a_{2} b_{2} c_{1}}\right. \\
& -\bar{\psi}^{a_{1} b_{1} c_{1}} \bar{\psi}^{a_{2} b_{1} c_{2}} \psi^{a_{1} b_{2} c_{2}} \psi^{a_{2} b_{2} c_{1}} \\
& \left.+\bar{\psi}^{a_{1} b_{1} c_{1}} \bar{\psi}^{a_{2} b_{2} c_{1}} \psi^{a_{1} b_{2} c_{2}} \psi^{a_{2} b_{1} c_{2}}\right) .
\end{aligned}
$$

This Hamiltonian is different from that of the $S U(N)^{2} \times O(N) \times U(1)$ symmetric complex tensor model [6]; which involves taking only the first term in Eq. (2.18).

\section{B. Feynman rules and two-point functions}

At first we list the Feynman rules which follow from the Hamiltonian (1.2). In Figs. 2 and 3, we define the propagators and interaction vertices for the given twoflavor tensor model. Since the interaction terms have a tetrahedral tensor structure, the melonic Feynman diagrams dominate in the large- $N$ limit. Let us define bare two-point functions

$$
\begin{aligned}
& G_{11}\left(\tau_{12}\right)=\frac{1}{N^{3}}\left\langle T \psi_{1}^{I}\left(\tau_{1}\right) \psi_{1}^{I}\left(\tau_{2}\right)\right\rangle_{0}, \\
& G_{22}\left(\tau_{12}\right)=\frac{1}{N^{3}}\left\langle T \psi_{2}^{I}\left(\tau_{1}\right) \psi_{2}^{I}\left(\tau_{2}\right)\right\rangle_{0},
\end{aligned}
$$

where the sum over indices $I$ is assumed. The leading melonic correction to the full two-point function $\mathbf{G}_{11}$ is represented in Fig. 4. Using that

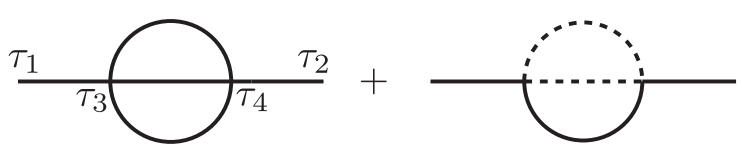

FIG. 4. The leading melonic correction to the full two-point function $\mathbf{G}_{11}$.

$$
J_{I J K L} J_{I J K L}=g^{2}\left(6 N^{6}-18 N^{4}+12 N^{3}\right),
$$

we find

$$
\begin{aligned}
\mathbf{G}_{11}\left(\tau_{12}\right)= & G_{11}\left(\tau_{12}\right)+g^{2} N^{3} \int d \tau_{3} d \tau_{4} G_{11}\left(\tau_{13}\right) \\
& \times\left[G_{11}\left(\tau_{34}\right)^{3}+3 \alpha^{2} G_{11}\left(\tau_{34}\right) G_{22}\left(\tau_{34}\right)^{2}\right] \\
& \times G_{11}\left(\tau_{42}\right)+\cdots
\end{aligned}
$$

A similar expression can be derived for $\mathbf{G}_{22}$. Since there is a symmetry $\psi_{1} \leftrightarrow \psi_{2}$, we can assume that $\mathbf{G}_{11}=\mathbf{G}_{22}=\mathbf{G}$ and obtain a Schwinger-Dyson equation for the full twopoint function (see Fig. 5)

$$
\begin{aligned}
\mathbf{G}\left(\tau_{12}\right)= & G\left(\tau_{12}\right)+\left(1+3 \alpha^{2}\right) g^{2} N^{3} \\
& \times \int d \tau_{3} d \tau_{4} G\left(\tau_{13}\right) \mathbf{G}\left(\tau_{34}\right)^{3} \mathbf{G}\left(\tau_{42}\right),
\end{aligned}
$$

where $G(\tau)=\frac{1}{2} \operatorname{sgn}(\tau)$ is the bare propagator.

In writing this Schwinger-Dyson equation, we implicitly make an important assumption that the two-point functions

$$
\begin{aligned}
& \mathbf{G}_{12}\left(\tau_{12}\right)=\frac{1}{N^{3}}\left\langle T \psi_{1}^{I}\left(\tau_{1}\right) \psi_{2}^{I}\left(\tau_{2}\right)\right\rangle \\
& \mathbf{G}_{21}\left(\tau_{12}\right)=\frac{1}{N^{3}}\left\langle T \psi_{2}^{I}\left(\tau_{1}\right) \psi_{1}^{I}\left(\tau_{2}\right)\right\rangle
\end{aligned}
$$

are zero $\mathbf{G}_{12}(\tau)=\mathbf{G}_{21}(\tau)=0$, due to the $\mathbb{Z}_{2}$ symmetry $\psi_{2} \rightarrow-\psi_{2}$. As we see below, the $\mathbb{Z}_{2}$ symmetry can be spontaneously broken for some range of parameter $\alpha$ and dimensionless coupling $\beta J$, where $\beta=1 / T$ is the inverse temperature and $J^{2}=g^{2} N^{3}$ is the effective coupling constant.

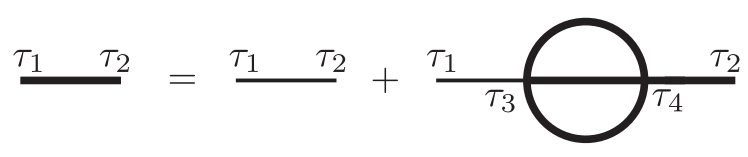

FIG. 5. Schwinger-Dyson equation for the full two-point function $\mathbf{G}\left(\tau_{12}\right)$. 
Let us first assume that $\mathbb{Z}_{2}$ symmetry is not broken and analyze the Schwinger-Dyson (SD) equation (2.22). At large coupling constant $\beta J$ and intermediate distances $1 / J \ll \tau \ll \beta$, the solution to this equation is given by

$$
\mathbf{G}(\tau)=\left(\frac{1}{4 \pi\left(1+3 \alpha^{2}\right)}\right)^{\frac{1}{4}} \frac{\operatorname{sgn}(\tau)}{|J \tau|^{1 / 2}} .
$$

\section{Scaling dimensions of bilinear operators}

We can use the large- $N$ Schwinger-Dyson equations for the three-point functions to deduce the scaling dimensions of four families of bilinear operators:

$$
\begin{aligned}
O_{1}^{2 n+1} & =\psi_{1} \partial_{\tau}^{2 n+1} \psi_{1}+\psi_{2} \partial_{\tau}^{2 n+1} \psi_{2}, \\
O_{2}^{2 n+1} & =\psi_{1} \partial_{\tau}^{2 n+1} \psi_{1}-\psi_{2} \partial_{\tau}^{2 n+1} \psi_{2}, \\
O_{3}^{2 n+1} & =\psi_{1} \partial_{\tau}^{2 n+1} \psi_{2}+\psi_{2} \partial_{\tau}^{2 n+1} \psi_{1}, \\
O_{4}^{2 n} & =\psi_{1} \partial_{\tau}^{2 n} \psi_{2}-\psi_{2} \partial_{\tau}^{2 n} \psi_{1},
\end{aligned}
$$

where $n=0,1,2, \ldots$, and the sum over tensor indices is assumed. [69] Each of these operators is invariant under the $O(N)^{3}$ symmetry, but they are distinguished by their transformations to discrete symmetry.

We take some operator $O(\tau)$ and consider two threepoint functions of the form

$$
\begin{aligned}
& v_{11}\left(\tau_{1}, \tau_{2}, \tau_{0}\right)=\left\langle\psi_{1}^{I}\left(\tau_{1}\right) \psi_{1}^{I}\left(\tau_{2}\right) O\left(\tau_{0}\right)\right\rangle, \\
& v_{22}\left(\tau_{1}, \tau_{2}, \tau_{0}\right)=\left\langle\psi_{2}^{I}\left(\tau_{1}\right) \psi_{2}^{I}\left(\tau_{2}\right) O\left(\tau_{0}\right)\right\rangle,
\end{aligned}
$$

where we assume summation over the index $I$. In the large$N$ limit, the functions (2.26) obey the melonic BetheSalpeter equations. They are schematically represented in Fig. 6. In the conformal limit, one can ignore the first diagram on the right and obtain

$$
\left(\begin{array}{l}
v_{11} \\
v_{22}
\end{array}\right)=\left(\begin{array}{ll}
K_{11,11} & K_{11,22} \\
K_{22,11} & K_{22,22}
\end{array}\right) *\left(\begin{array}{c}
v_{11} \\
v_{22}
\end{array}\right)
$$

where assuming that $\mathbf{G}_{11}=\mathbf{G}_{22}=\mathbf{G}$, we find

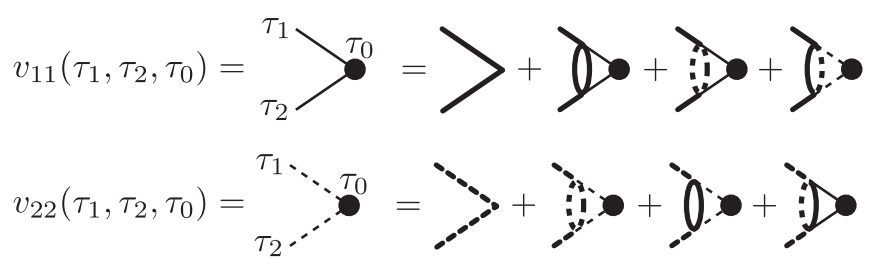

FIG. 6. The Bethe-Salpeter equations for the three-point functions $v_{11}$ and $v_{22}$.

$$
\begin{aligned}
& K_{11,11}=K_{22,22}=\frac{1+\alpha^{2}}{1+3 \alpha^{2}} K_{c}, \\
& K_{11,22}=K_{22,11}=\frac{2 \alpha^{2}}{1+3 \alpha^{2}} K_{c},
\end{aligned}
$$

and $K_{c}$ is the kernel of the SYK model defined in conformal limit as

$K_{c}\left(\tau_{1}, \tau_{2} ; \tau_{3}, \tau_{4}\right)=-\frac{3}{4 \pi} \frac{\operatorname{sgn}\left(\tau_{13}\right) \operatorname{sgn}\left(\tau_{24}\right)}{\left|\tau_{13}\right|^{2 \Delta}\left|\tau_{24}\right|^{2 \Delta}\left|\tau_{34}\right|^{2-4 \Delta}}, \quad \Delta=\frac{1}{4}$

An arbitrary conformal three-point function of the form (2.26) with an operator of scaling dimension $h$ has the form

$$
v_{h}\left(\tau_{1}, \tau_{2}, \tau_{0}\right)=\frac{c \operatorname{sgn}\left(\tau_{12}\right)}{\left|\tau_{01}\right|^{h}\left|\tau_{02}\right|^{h}\left|\tau_{12}\right|^{2 \Delta-h}},
$$

and obviously must be antisymmetric under $\tau_{1} \leftrightarrow \tau_{2}$. This three-point function is an eigenvector of the kernel $K_{c}$ with the eigenvalue $g(h):[70]$

$g(h) \int d \tau_{3} d \tau_{4} K_{c}\left(\tau_{1}, \tau_{2} ; \tau_{3}, \tau_{4}\right) v_{h}\left(\tau_{3}, \tau_{4}, \tau_{0}\right)=v_{h}\left(\tau_{1}, \tau_{2}, \tau_{0}\right)$

To solve Eq. (2.27), one has to find eigenvalues of the matrix and equate them to unity, thus obtaining an equation for possible scaling dimensions. It easy to see that this matrix acquires a diagonal form in the basis of vectors $v_{11}+v_{22}$ and $v_{11}-v_{22}$, and we find two equations for the scaling dimensions

$$
\begin{aligned}
& g_{A}(h)=1, \quad \frac{1-\alpha^{2}}{1+3 \alpha^{2}} g_{A}(h)=1, \\
& g_{A}(h)=-\frac{3}{2} \frac{\tan \left[\frac{\pi}{2}\left(h-\frac{1}{2}\right)\right]}{h-1 / 2} .
\end{aligned}
$$

The scaling dimensions of the operator $O_{1}^{2 n+1}=$ $\psi_{1} \partial_{\tau}^{2 n+1} \psi_{1}+\psi_{2} \partial_{\tau}^{2 n+1} \psi_{2}$ satisfy $g_{A}(h)=1$ and are independent of $\alpha$. They are given by the well-known series $h=2.00,3.77,5.68,7.63,9.60, \ldots$, which approaches $2 n+\frac{3}{2}$. These are the same scaling dimensions as in the basic $O(N)^{3}$ tensor model [6] and the SYK model. On the other hand, the scaling dimensions of operators $O_{2}^{2 n+1}=$ $\psi_{1} \partial_{\tau}^{2 n+1} \psi_{1}-\psi_{2} \partial_{\tau}^{2 n+1} \psi_{2}$ are given by $\left[\left(1-\alpha^{2}\right) /\left(1+3 \alpha^{2}\right)\right]$ $g_{A}(h)=1$ and depend on $\alpha$. As a check, we note that for $\alpha=0$ the spectra of $O_{2}$ and $O_{1}$ are the same; this is as expected since the two flavors are decoupled.

Now consider the last possible three-point function

$$
v_{12}\left(\tau_{1}, \tau_{2}, \tau_{0}\right)=\left\langle\psi_{1}^{I}\left(\tau_{1}\right) \psi_{2}^{I}\left(\tau_{2}\right) O\left(\tau_{0}\right)\right\rangle .
$$




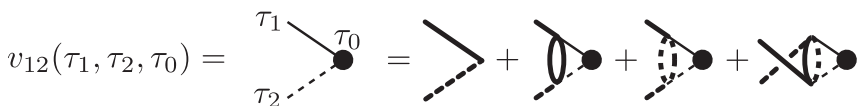

FIG. 7. The Bethe-Salpeter equations for the three-point functions $v_{12}$.

The melonic Bethe-Salpeter equation for this three-point function is represented in Fig. 7, and in the conformal limit, neglecting the first diagram on the right, we get

$$
\begin{aligned}
v_{12}\left(\tau_{1}, \tau_{2}, \tau_{0}\right)= & \int d \tau_{3} d \tau_{4} \frac{2}{1+3 \alpha^{2}}\left[\alpha K_{c}\left(\tau_{1}, \tau_{2} ; \tau_{3}, \tau_{4}\right)\right. \\
& \left.-\alpha^{2} K_{c}\left(\tau_{1}, \tau_{2} ; \tau_{4}, \tau_{3}\right)\right] v_{12}\left(\tau_{3}, \tau_{4}, \tau_{0}\right)
\end{aligned}
$$

In this case, there are two general possibilities for the conformal three-point function, namely, the antisymmetric and symmetric cases

$$
\begin{aligned}
& v_{h}^{A}\left(\tau_{1}, \tau_{2}, \tau_{0}\right)=\frac{c \operatorname{sgn}\left(\tau_{12}\right)}{\left|\tau_{01}\right|^{h}\left|\tau_{02}\right|^{h}\left|\tau_{12}\right|^{2 \Delta-h}}, \\
& v_{h}^{S}\left(\tau_{1}, \tau_{2}, \tau_{0}\right)=\frac{c \operatorname{sgn}\left(\tau_{01}\right) \operatorname{sgn}\left(\tau_{02}\right)}{\left|\tau_{01}\right|^{h}\left|\tau_{02}\right|^{h}\left|\tau_{12}\right|^{2 \Delta-h}} .
\end{aligned}
$$

Therefore, we find equations which determine the spectra of antisymmetric and symmetric operators

$$
\begin{aligned}
& \frac{2\left(\alpha+\alpha^{2}\right)}{1+3 \alpha^{2}} g_{A}(h)=1, \quad \frac{6\left(\alpha-\alpha^{2}\right)}{1+3 \alpha^{2}} g_{S}(h)=1, \\
& g_{S}(h)=-\frac{1}{2} \frac{\tan \left[\frac{\pi}{2}\left(h+\frac{1}{2}\right)\right]}{h-1 / 2} .
\end{aligned}
$$

The scaling dimensions of operators $O_{3}^{2 n+1}$ satisfy the first equation above and $O_{4}^{2 n}$ the second. We can check this result by comparing with the results for the complex bipartite fermion model (2.16). It was found [17] that the scaling dimensions of $O_{4}^{2 n}$ are determined by

$$
g_{\mathrm{sym}}(h)=\frac{3}{2} \frac{\tan \left[\frac{\pi}{2}\left(h+\frac{1}{2}\right)\right]}{h-1 / 2}=1,
$$

and indeed for $\alpha=-1$ we get $\left\{\left[6\left(\alpha-\alpha^{2}\right)\right] /\left(1+3 \alpha^{2}\right)\right\} g_{S}$ $(h)=g_{\text {sym }}(h)$.

To summarize, we find that scaling dimensions of the operators (2.25) can be obtained by solving equations $g_{i}(h)=1$, where

$$
\begin{aligned}
& {\left[g_{1}(h), g_{2}(h), g_{3}(h), g_{4}(h)\right]} \\
& =\left(g_{A}(h), \frac{1-\alpha^{2}}{1+3 \alpha^{2}} g_{A}(h), \frac{2 \alpha(1+\alpha)}{1+3 \alpha^{2}} g_{A}(h), \frac{6 \alpha(1-\alpha)}{1+3 \alpha^{2}} g_{S}(h)\right) .
\end{aligned}
$$

The duality relation (2.11) is reflected in the behavior of functions $g_{i}(h)$, which define scaling dimensions of the operators $O_{i}$. Using Eqs. (2.38) and (2.11), one finds

$$
\left[g_{1}(h), g_{2}(h), g_{3}(h), g_{4}(h)\right] \rightarrow\left[g_{1}(h), g_{3}(h), g_{2}(h), g_{4}(h)\right]
$$

Indeed, under $\psi \rightarrow \tilde{\psi}$, the operators $O_{i}$ transform as $\left(O_{1}, O_{2}, O_{3}, O_{4}\right) \rightarrow\left(O_{1}, O_{3}, O_{2}, O_{4}\right)$.

\section{Complex scaling dimensions}

In this section, we examine if there exist any complex solutions of the equations $g_{i}(h)=1$ defined in Eq. (2.38). If such a complex root exists, then a conformal primary has a complex scaling dimension, which leads to a destabilization of the model. Indeed, a complex scaling dimension of the form $\frac{1}{2} \pm$ if corresponds to a scalar fields in $\mathrm{AdS}_{2}$ whose $m^{2}$ is below the Breitenlohner-Freedman bound

$$
\begin{gathered}
m_{\mathrm{BF}}^{2}=-\frac{1}{4} . \text { Since } \Delta=\frac{1}{2} \pm \sqrt{\frac{1}{4}+m^{2}}[53-55], \\
m^{2}=-\frac{1}{4}-f^{2}<m_{\mathrm{BF}}^{2} .
\end{gathered}
$$

In such a case, one may expect "tachyon condensation" in AdS space. In the dual CFT, the operator dual to the tachyon acquires an expectation value leading to symmetry breaking. We obtain some support for this picture.

First of all, we notice that the functions $g_{A}(h)$ and $g_{S}(h)$ are real only if $h$ is real or $h=\frac{1}{2}+i f$ for real $f$. Next, it is easy to check that

$-\frac{3 \pi}{4} \leq g_{A}(1 / 2+i f)<0, \quad-\infty \leq g_{S}(1 / 2+i f)<0$.

Using the fact that $-\frac{1}{3} \leq\left[\left(1-\alpha^{2}\right) /\left(1+3 \alpha^{2}\right)\right] \leq 1$ (and the same for $[2 \alpha(1+\alpha)] /\left(1+3 \alpha^{2}\right)$ due to duality), we conclude that equations $g_{i}(1 / 2+i f)=1$ for $i=1,2,3$ do not have solutions; thus, the scaling dimensions of the operators $\mathrm{O}_{1}, \mathrm{O}_{2}$, and $\mathrm{O}_{3}$ are always real.

On the other hand, since $\left\{[6 \alpha(1-\alpha)] /\left(1+3 \alpha^{2}\right)\right\}<0$ for negative $\alpha$, the equation $g_{4}(h)=1$ has solutions $h=1 / 2 \pm i f(\alpha)$, where $f(\alpha)$ can be found from the equation

$$
f \tanh (\pi f / 2)=-\frac{3 \alpha(1-\alpha)}{1+3 \alpha^{2}} .
$$

The plot of $f(\alpha)$ is shown in Fig. 8. For slightly negative $\alpha$, we find

$$
f(\alpha)=\sqrt{\frac{-6 \alpha}{\pi}}[1+O(\alpha)]
$$




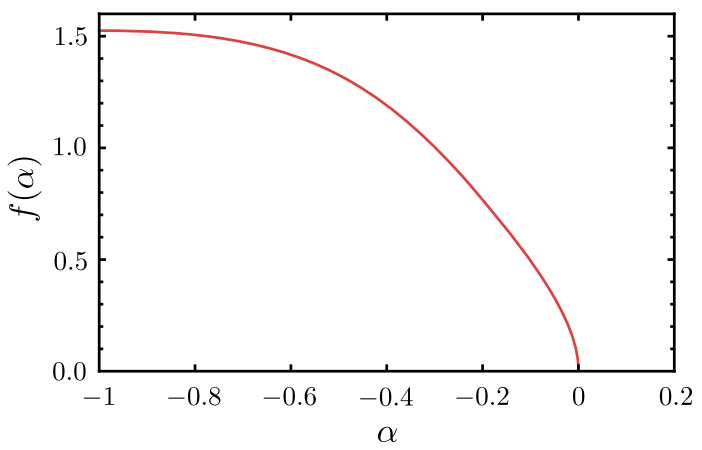

FIG. 8. Imaginary part of the scaling dimension of the fermion number operator $Q$. At $\alpha=-1$, it reaches its maximum value of approximately 1.5251 .

while $f(-1) \approx 1.5251$ in agreement with the result for the bipartite model found in Ref. [17]. Thus, for $-1 \leq \alpha<0$ there is an operator with the complex scaling dimension $h=1 / 2+i f(\alpha)$ or its complex conjugate: the fermion number operator $Q=i O_{4}^{0}$. The complex scaling dimension makes the conformal large- $N$ limit unstable.

For $0 \leq \alpha \leq 1 / 3$, there are no complex solutions of $g_{4}(h)=1$. The two lowest positive real solutions $h_{ \pm}(\alpha)$ satisfy $h_{+}+h_{-}=1$. These two roots are the scaling dimensions of operator $Q$ in two different large- $N$ CFTs [71], as we explain below. We find

$$
h_{+}(\alpha)=1 / 2+\sqrt{\frac{6 \alpha}{\pi}}+\cdots
$$

when $\alpha$ is small and positive, and $h_{+}(1 / 3)=1$. The fact that $\alpha=0$ is the lower edge of the conformal window is related to the behavior of the scaling dimension of the "double-trace" operator $Q^{2}$. In the large- $N$ limit, $\Delta_{Q^{2}}=$ $2 \Delta_{Q}$. In one of the CFTs, $\Delta_{Q}=h_{+}$so that $\Delta_{Q^{2}}>1$. Since the operator $Q^{2}$ is irrelevant, this CFT is stable. There is Renormalization Group flow leading to it, which originates from another large- $N$ fixed point where $Q^{2}$ is relevant [72,73]. At this UV fixed point, $\Delta_{Q}=h_{-}$. When $\alpha=0$, the two fixed points merge and annihilate, as in various other theories, e.g., Refs. [48,49,57,58]. For $-1 \leq \alpha<0$, there are two different theories containing complex dimension $\Delta_{Q}=1 / 2+i f(\alpha)$ or its complex conjugate. They may be formally regarded as "complex CFTs" [52], but we see in the next section that their true physics includes symmetry breaking, which leads to a gap in the energy spectrum.

\section{SYMMETRY BREAKING}

In Sec. II D, we show that for the coupled tensor model (1.2) in the range $-1 \leq \alpha<0$, the fermion number operator $Q=i \psi_{1}^{I} \psi_{2}^{I}$ has a complex scaling dimension, signaling an instability of the conformal phase of the model. In this section, we show that this operator acquires a vacuum expectation value (VEV) in the true low-temperature phase of the large- $N$ model. It is, therefore, tempting to make the following conjecture.

Conjecture. If the assumption of conformal invariance in a large- $N$ theory leads to a single-trace operator with a complex scaling dimension of the form $d / 2+i f$, then in the true low-temperature phase, this operator acquires a VEV.

In our case, the $O(N)^{3}$ symmetry implies that

$$
\left\langle i \psi_{1}^{I} \psi_{2}^{J}\right\rangle=\delta^{I J} A,
$$

where we use the shorthand notation $I=a b c$, and $A$ is of order 1 in the large- $N$ limit.

This VEV leads to an exponential decay of correlation functions and signifies a gap in the energy spectrum. Furthermore, the VEV (3.1) implies that various discrete symmetries, including the particle-hole symmetry (2.6), the interchange symmetry between $\psi_{1}, \psi_{2}$, and the reflection symmetry $\psi_{2} \rightarrow-\psi_{2}$, are spontaneously broken. Therefore, one should expect a second-order phase transition between the broken and unbroken symmetry phases. In addition, the spontaneously broken symmetry also implies a ground-state degeneracy in the large- $N$ energy spectrum [74].

In this section, we extensively analyze the phenomenon of symmetry breaking, sometimes using the SYK counterpart (1.1) of the $O(N)^{3}$ tensor model (1.2). The two models have many similarities at large $N$ : They share the same Schwinger-Dyson equations and the spectra of bilinear operators. The SYK formulation, however, is advantageous for the purpose of exact numerical diagonalizations: We can study cases where the integer $N_{\mathrm{SYK}}$ is not the cube of an integer.

Let us first demonstrate the connection between the tensor model and the SYK counterpart. For the one-flavor $O(N)^{3}$ tensor model, the analogous SYK model has the random tensor $J_{i j k l}$ which is fully antisymmetric. The mixed term $A_{i j k l} \chi_{1}^{i} \chi_{1}^{j} \chi_{2}^{k} \chi_{2}^{l}$ has only the symmetries

$$
A_{i j k l}=-A_{j i k l}=-A_{i j l k}=A_{k l i j},
$$

which are the same as for the Riemann tensor. However, the full interaction term following from Eq. (1.2) is

$$
\begin{gathered}
A_{i j k l}\left(\chi_{1}^{i} \chi_{1}^{j} \chi_{2}^{k} \chi_{2}^{l}+\chi_{1}^{i} \chi_{2}^{j} \chi_{1}^{k} \chi_{2}^{l}+\chi_{1}^{i} \chi_{2}^{j} \chi_{2}^{k} \chi_{1}^{l}\right) \\
=\left(A_{i j k l}+A_{i l j k}+A_{i k l j}\right) \chi_{1}^{i} \chi_{1}^{j} \chi_{2}^{k} \chi_{2}^{l} .
\end{gathered}
$$

Since $A_{i j k l}+A_{i l j k}+A_{i k l j}$ is fully antisymmetric due to Eq. (3.2), the mixed term has a fully antisymmetric random coupling. We assume that it is proportional to the coupling in the diagonal term of Eq. (1.2), and are thus led to the random model (1.1). This model is the special $M=2$ case of a periodic SYK chain model 
$H_{\text {chain }}=\frac{1}{4 !} J_{i j k l} \sum_{x=1}^{M}\left(\chi_{x}^{i} \chi_{x}^{j} \chi_{x}^{k} \chi_{x}^{l}+3 \alpha \chi_{x}^{i} \chi_{x}^{j} \chi_{x+1}^{k} \chi_{x+1}^{l}\right)$,

where the integer $x$ labels the lattice site, and $\chi_{M+1}^{i} \equiv \chi_{1}^{i}$. This Hamiltonian can be obtained from the model of Ref. [26] by identifying the separate random couplings up to a factor of $\alpha$.

Introducing the complex combination $\psi^{j}=(1 / \sqrt{2})$ $\left(\chi_{1}^{j}+i \chi_{2}^{j}\right)$, we may write the Hamiltonian (1.1) as

$$
\begin{aligned}
H= & \frac{1}{4 !} J_{i j k l}\left(\frac{1-3 \alpha}{2}\left(\psi^{i} \psi^{j} \psi^{k} \psi^{l}+\bar{\psi}^{i} \bar{\psi}^{j} \bar{\psi}^{k} \bar{\psi}^{l}\right)\right. \\
& \left.+3(1+\alpha) \bar{\psi}^{i} \bar{\psi}^{j} \psi^{k} \psi^{l}\right) .
\end{aligned}
$$

As usual, we assume that each variable $J_{i j k l}$ has a Gaussian distribution with variance $6 J^{2} N_{\text {SYK }}^{-3}$. We typically state energies in units of $J$ or equivalently set $J=1$.

The duality symmetry described in Sec. II A applies to the coupled SYK model (1.1) and again allows us to restrict $\alpha$ to the range from -1 to $1 / 3$. There are two interesting limiting cases. For $\alpha=-1$, the transformation (2.11) maps $H \rightarrow-H$. This means that for any random choice of $J_{i j k l}$, the energy spectrum is exactly symmetric under $E \rightarrow-E$. This can be seen in the histograms of the spectrum shown in Fig. 18; in particular, there are many states whose energy is exactly zero. For $\alpha=-1$, the model is a random counterpart of the complex bipartite model:

$$
H_{\alpha=-1}=\frac{2}{4 !} J_{i j k l}\left(\psi^{i} \psi^{j} \psi^{k} \psi^{l}+\bar{\psi}^{i} \bar{\psi}^{j} \bar{\psi}^{k} \bar{\psi}^{l}\right) .
$$

The fermion number operator

$$
Q=i \chi_{1}^{j} \chi_{2}^{j}=\frac{1}{2}\left[\bar{\psi}^{j}, \psi^{j}\right]
$$

does not in general commute with $H$, but it is conserved mod 4, just like in the Maldacena-Qi model [45]. For $\alpha=1 / 3$, however, we find the Hamiltonian

$$
H_{\alpha=\frac{1}{3}}=\frac{4}{4 !} J_{i j k l} \bar{\psi}^{i} \bar{\psi}^{j} \psi^{k} \psi^{l}, \quad\left[Q, H_{\alpha=\frac{1}{3}}\right]=0 .
$$

Thus, there is an enhanced $U(1)$ symmetry $\psi^{j} \rightarrow e^{i \gamma} \psi^{j}$ [75]. We note that for $\alpha=1 / 3$, the scaling dimension of operator $Q=O_{4}^{0}$ is $h=1$, consistent with charge conservation. Also, here $g_{2}(h)=g_{3}(h)$ so that the scaling dimensions of $O_{2}^{2 n+1}$ and $O_{3}^{2 n+1}$ are equal. This is because

$$
O_{2}^{2 n+1}+i O_{3}^{2 n+1}=2 \psi^{j} \partial_{t}^{2 n+1} \psi^{j} .
$$

Furthermore, the transformation (2.11) maps $H_{\alpha=\frac{1}{3}}$ into itself, so the theory is self-dual.
For general $\alpha$, the model (1.1) has multiple discrete symmetries, which are discussed in more detail in Appendix A. These discrete symmetries can be spontaneously broken due to a VEV of $Q$ if $Q$ is not invariant under them. In the model (1.1), there are two symmetries that are not broken by a VEV of $Q$ : the antiunitary timereversal symmetry $K$ and a $\mathbb{Z}_{4}$ symmetry generated by $\pi / 2$ rotation $R$ in $\chi_{1}, \chi_{2}$,

$$
R \chi_{1} R^{\dagger}=\chi_{2}, \quad R \chi_{2} R^{\dagger}=-\chi_{1} .
$$

They both preserve $Q$. The model (1.1) also has multiple reflection symmetries that are spontaneously broken by the VEV of $Q$, which we list in Appendix A. In fact, all unitary discrete symmetries of the model (1.1) form the dihedral group of order $8, D_{4}$. In our case, any two broken symmetries that can be related by an unbroken symmetry do not produce extra ground-state degeneracy, and therefore, it is enough to focus on one of them.

Let us focus on the particle-hole symmetry [37-42] generated by

$$
\mathcal{P}=K \prod_{i=1}^{N_{\mathrm{SYK}}}\left(\psi^{i}+\bar{\psi}^{i}\right), \quad \mathcal{P}^{2}=(-1)^{N_{\mathrm{SYK}}\left(N_{\mathrm{SYK}}-1\right) / 2} .
$$

It acts on the fermion number as

$$
\mathcal{P} Q \mathcal{P}=-\mathcal{P}^{2} Q
$$

For $N_{\mathrm{SYK}}$ not divisible by 4 , there is a twofold degeneracy of the ground state in Sec. III C due to an anomaly in the particle-hole symmetry [37-42]. For $N_{\text {SYK }}$ divisible by 4 , this symmetry is not anomalous, and we find a nondegenerate ground state, which is followed by a nearby state when $-1 \leq \alpha<0$. The two lowest states become degenerate in the large- $N_{\mathrm{SYK}}$ limit, and they are separated by a gap from the remaining states. This asymptotic degeneracy leads to a spontaneous symmetry breaking through the formation of an expectation value of $Q$. We demonstrate this effect by solving the large- $N_{\text {SYK }}$ Schwinger-Dyson equations for the Green functions and with diagonalizations at finite $N_{\mathrm{SYK}}$.

\section{A. Schwinger-Dyson equations and the effective action}

In this section, we derive the large- $N_{\mathrm{SYK}}$ effective action of $G \Sigma$ type and the Schwinger-Dyson equations for the coupled SYK model (1.1). Following Ref. [44], we introduce bilocal variables

$$
G_{a b}\left(\tau, \tau^{\prime}\right)=\frac{1}{N_{\mathrm{SYK}}}\left\langle T \chi_{a}^{i}(\tau) \chi_{b}^{i}\left(\tau^{\prime}\right)\right\rangle
$$

and the corresponding Lagrange multipliers $\Sigma_{a b}\left(\tau, \tau^{\prime}\right)$, where $a, b=1,2$. The effective action is given by 


$$
\begin{aligned}
-\frac{\beta S_{\mathrm{eff}}}{N_{\mathrm{SYK}}}= & \log \operatorname{Pf}\left(\partial_{\tau} \delta_{a b}-\Sigma_{a b}\right)-\frac{1}{2} \int d \tau d \tau^{\prime}\left[\sum_{a, b} \Sigma_{a b}\left(\tau, \tau^{\prime}\right) G_{a b}\left(\tau, \tau^{\prime}\right)-\frac{J^{2}}{4}\left(\sum_{a, b} G_{a b}^{4}\left(\tau, \tau^{\prime}\right)\right.\right. \\
& +6 \alpha\left[G_{12}^{2}\left(\tau, \tau^{\prime}\right)+G_{21}^{2}\left(\tau, \tau^{\prime}\right)\right]\left[G_{11}^{2}\left(\tau, \tau^{\prime}\right)+G_{22}^{2}\left(\tau, \tau^{\prime}\right)\right]+6 \alpha^{2}\left[G_{11}^{2}\left(\tau, \tau^{\prime}\right) G_{22}^{2}\left(\tau, \tau^{\prime}\right)\right. \\
& \left.\left.\left.+G_{12}^{2}\left(\tau, \tau^{\prime}\right) G_{21}^{2}\left(\tau, \tau^{\prime}\right)+4 G_{11}\left(\tau, \tau^{\prime}\right) G_{22}\left(\tau, \tau^{\prime}\right) G_{12}\left(\tau, \tau^{\prime}\right) G_{21}\left(\tau, \tau^{\prime}\right)\right]\right)\right]
\end{aligned}
$$

By translation invariance,

$G_{a b}\left(\tau, \tau^{\prime}\right)=G_{a b}\left(\tau-\tau^{\prime}\right), \quad \Sigma_{a b}\left(\tau, \tau^{\prime}\right)=\Sigma_{a b}\left(\tau-\tau^{\prime}\right)$.

We also have the general properties

$$
\begin{aligned}
& G_{11}(\tau)=-G_{11}(-\tau), \quad G_{22}(\tau)=-G_{22}(-\tau), \\
& G_{12}(\tau)=-G_{21}(-\tau) .
\end{aligned}
$$

The SD equations for the two-point functions assume the form [76]

$$
\begin{aligned}
& \partial_{\tau} G_{11}(\tau)-\int d \tau^{\prime}\left[\Sigma_{11}\left(\tau-\tau^{\prime}\right) G_{11}\left(\tau^{\prime}\right)+\Sigma_{12}\left(\tau-\tau^{\prime}\right) G_{21}\left(\tau^{\prime}\right)\right]=\delta(\tau), \\
& \partial_{\tau} G_{12}(\tau)-\int d \tau^{\prime}\left[\Sigma_{11}\left(\tau-\tau^{\prime}\right) G_{12}\left(\tau^{\prime}\right)+\Sigma_{12}\left(\tau-\tau^{\prime}\right) G_{22}\left(\tau^{\prime}\right)\right]=0, \\
& J^{-2} \Sigma_{11}=G_{11}^{3}+3 \alpha G_{11}\left(G_{12}^{2}+G_{21}^{2}\right)+3 \alpha^{2} G_{11} G_{22}^{2}+6 \alpha^{2} G_{22} G_{12} G_{21}, \\
& J^{-2} \Sigma_{12}=G_{12}^{3}+3 \alpha G_{12}\left(G_{11}^{2}+G_{22}^{2}\right)+3 \alpha^{2} G_{12} G_{21}^{2}+6 \alpha^{2} G_{11} G_{22} G_{21},
\end{aligned}
$$

and similarly for $1 \leftrightarrow 2$. These equations and the effective action are invariant under $1 \leftrightarrow 2$ and $G_{12} \rightarrow-G_{12}$, $G_{21} \rightarrow-G_{21}$.

\section{B. Solutions of Schwinger-Dyson equations and symmetry breaking}

For $0 \leq \alpha \leq 1 / 3$, there are no operators with complex scaling dimensions, so it is consistent to assume that the discrete symmetries are unbroken and set $G_{12}=0$ and $G_{11}=G_{22}$ to obtain a nearly conformal solution in the low-energy limit. However, the appearance of a complex scaling dimension for $-1 \leq \alpha<0$ shows that such a conformal phase is unstable. We show that in this range of $\alpha$, the true phase of the theory exhibits spontaneous symmetry breaking. In order to exhibit it, we have to allow the possibility that $G_{12}(\tau) \neq 0$. The underlying $\mathbb{Z}_{2}$ symmetry of the Hamiltonian (1.1) implies that such solutions must come in pairs related by $G_{12}(\tau) \rightarrow$ $-G_{12}(\tau)$ (in our numerical work, we typically exhibit only one of these two solutions). They correspond to working around the two inequivalent vacua, which we call $\left|0_{+}\right\rangle$and $\left|0_{-}\right\rangle$. They are distinguished by the sign of the expectation value of operator $Q=i \chi_{1}^{i} \chi_{2}^{i}$ :

$$
\begin{aligned}
& \left\langle 0_{+}|Q| 0_{+}\right\rangle=A N_{\mathrm{SYK}}, \quad\left\langle 0_{-}|Q| 0_{-}\right\rangle=-A N_{\mathrm{SYK}}, \\
& \left\langle 0_{-}|Q| 0_{+}\right\rangle=0
\end{aligned}
$$

The unbroken symmetry $R$ in Eq. (3.10) implies

$$
G_{12}(-\tau)=-G_{21}(\tau)=G_{12}(\tau), \quad G_{22}(\tau)=G_{11}(\tau),
$$

and similarly for $\Sigma_{a b}$. Using these constraints, we obtain for the effective action

$$
\begin{aligned}
-\frac{\beta S_{\mathrm{eff}}}{N_{\mathrm{SYK}}}= & \log \operatorname{Pf}\left(\delta_{a b} \partial_{\tau}-\Sigma_{a b}\right)-\beta \int_{0}^{\beta} d \tau\left(\Sigma_{11} G_{11}+\Sigma_{12} G_{12}\right. \\
& \left.-\frac{J^{2}}{4}\left[\left(1+3 \alpha^{2}\right)\left(G_{11}^{4}+G_{12}^{4}\right)+12 \alpha(1-\alpha) G_{11}^{2} G_{12}^{2}\right]\right) .
\end{aligned}
$$

The Schwinger-Dyson equations become

$$
\begin{gathered}
\partial_{\tau} G_{11}(\tau)-\int d \tau^{\prime}\left[\Sigma_{11}\left(\tau-\tau^{\prime}\right) G_{11}\left(\tau^{\prime}\right)\right. \\
\left.-\Sigma_{12}\left(\tau-\tau^{\prime}\right) G_{12}\left(\tau^{\prime}\right)\right]=\delta(\tau), \\
\partial_{\tau} G_{12}(\tau)-\int d \tau^{\prime}\left[\Sigma_{11}\left(\tau-\tau^{\prime}\right) G_{12}\left(\tau^{\prime}\right)\right. \\
\left.+\Sigma_{12}\left(\tau-\tau^{\prime}\right) G_{11}\left(\tau^{\prime}\right)\right]=0
\end{gathered}
$$



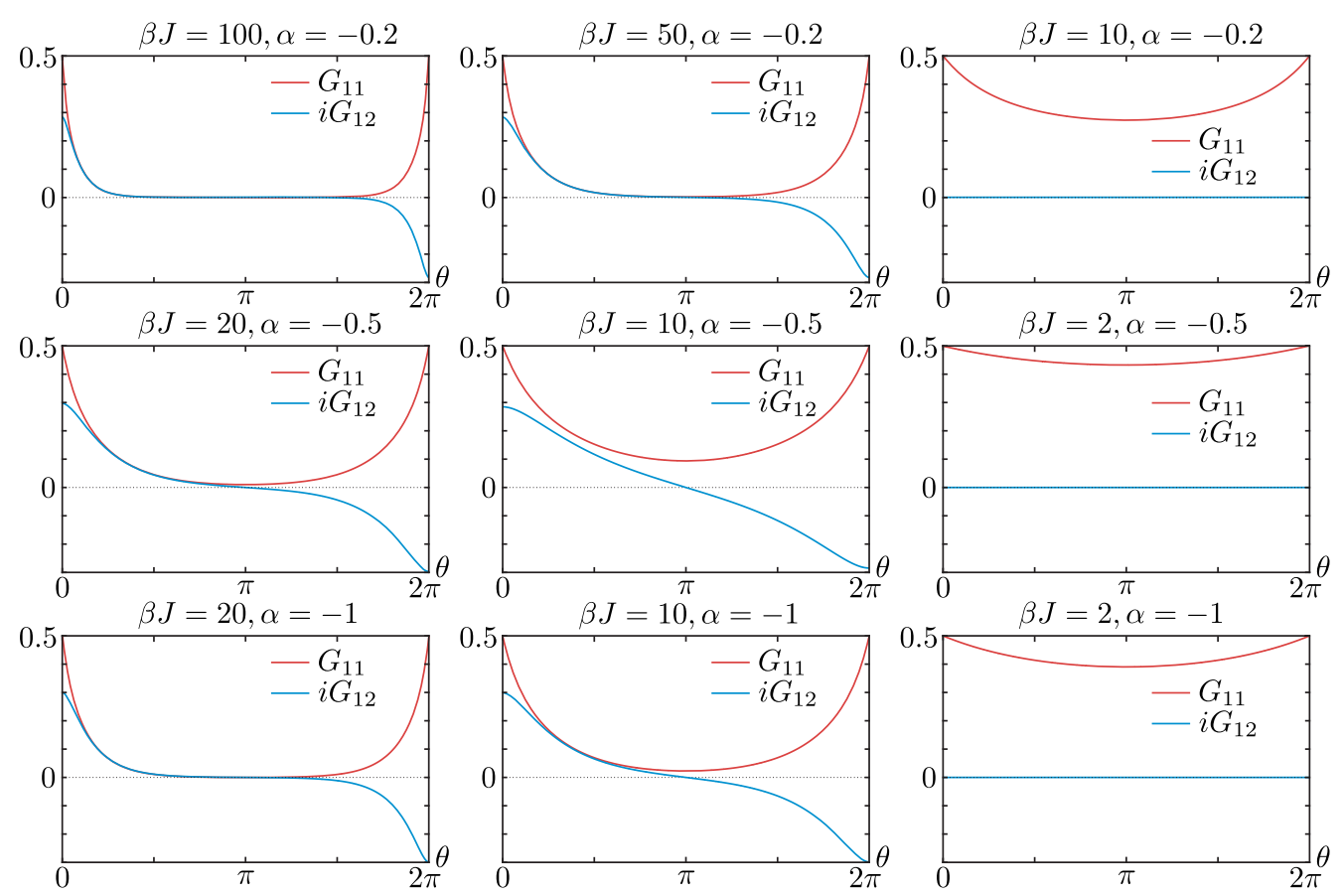

FIG. 9. Numerical solutions for $\alpha=-1,-0.5,-0.2$, and various values of $\beta J$.

and

$$
\begin{aligned}
& J^{-2} \Sigma_{11}(\tau)=\left(1+3 \alpha^{2}\right) G_{11}^{3}(\tau)+6 \alpha(1-\alpha) G_{11}(\tau) G_{12}^{2}(\tau), \\
& J^{-2} \Sigma_{12}(\tau)=\left(1+3 \alpha^{2}\right) G_{12}^{3}(\tau)+6 \alpha(1-\alpha) G_{11}^{2}(\tau) G_{12}(\tau) .
\end{aligned}
$$

Equation (3.20) may be written in momentum space as

$$
\begin{aligned}
& G_{11}\left(\omega_{n}\right)=\frac{-i \omega_{n}-\Sigma_{11}\left(\omega_{n}\right)}{\left(-i \omega_{n}-\Sigma_{11}\right)^{2}+\Sigma_{12}^{2}}, \\
& G_{12}\left(\omega_{n}\right)=\frac{\Sigma_{12}\left(\omega_{n}\right)}{\left(-i \omega_{n}-\Sigma_{11}\right)^{2}+\Sigma_{12}^{2}} .
\end{aligned}
$$

These equations together with Eq. (3.21) can be solved numerically using the method of weighted iterations used

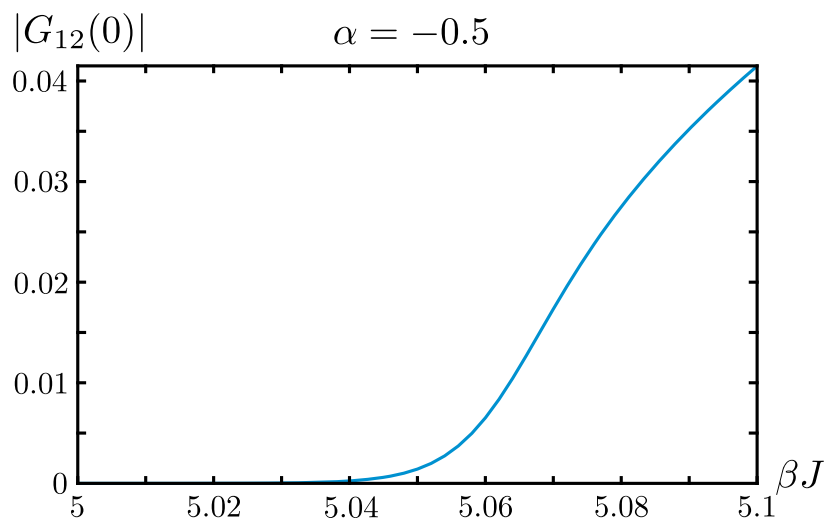

FIG. 10. The expectation value of $Q / N_{\text {SYK }}$, i.e., $\left|G_{12}(0)\right|$, as a function of $\beta J$ for $\alpha=-0.5$. The region near $(\beta J)_{\text {crit }}$ is shown. in Ref. [19,77]. To trigger the spontaneous symmetry breaking, we start our iteration process with a tiny nonzero $G_{12}(\tau)$ which is purely imaginary. If we are in the unbroken phase, after the iterations, $G_{12}$ becomes zero, whereas if we are in the broken phase, we find a nonzero purely imaginary solution for $G_{12}$.

The plots of $G_{11}$ and $i G_{12}$ for different values of $\alpha$ and $\beta J$ are shown in Fig. 9. For each value of $\alpha$ between -1 and 0 , there are two phases. In the low-temperature phase (large $\beta J)$, there are three distinct solutions: two solutions with nonvanishing $i G_{12}$ related by $G_{12}(\tau) \rightarrow-G_{12}(\tau)$ and the one where $G_{12}(\tau)=0$. The solutions with nonvanishing $i G_{12}$ are the ones with the lower free energy. As $\beta J$ decreases, $\left|G_{12}(\tau)\right|$ decreases everywhere for the nontrivial solution (see Figs. 9 and 10) and at the critical value

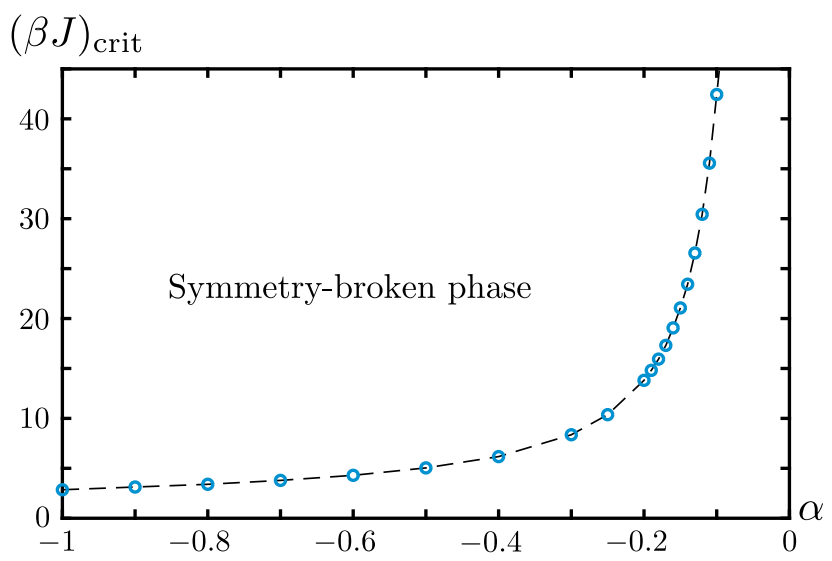

FIG. 11. Critical value of $\beta J$ as a function of $\alpha$. 

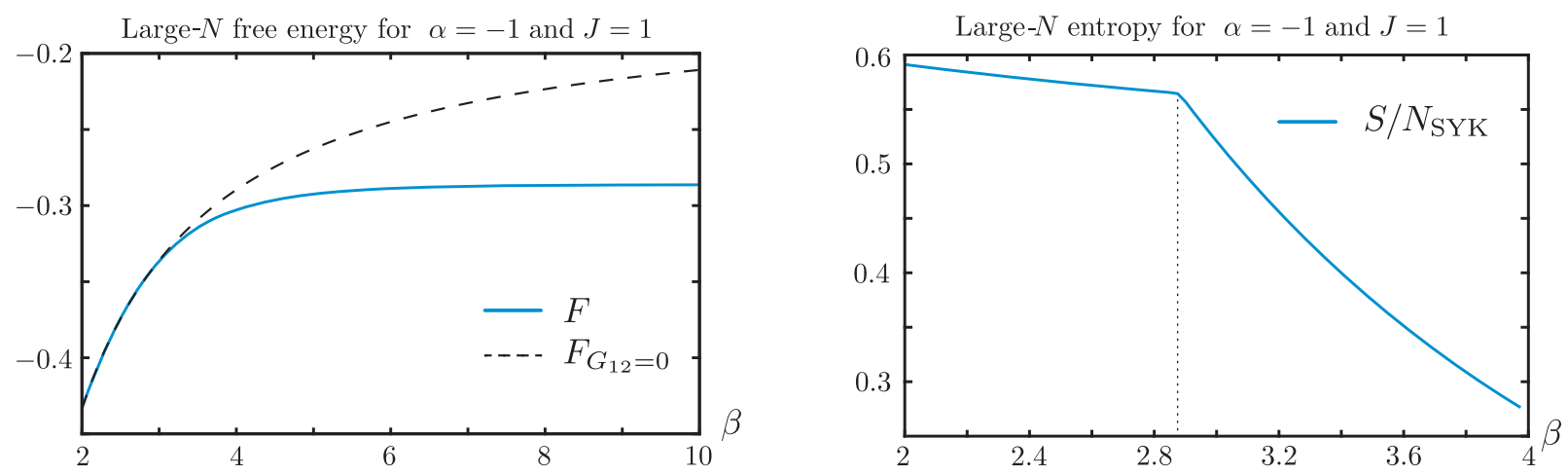

FIG. 12. Large- $N$ free energies of the true numerical solution and the solution with $G_{12}=0$ for $\alpha=-1, J=1$. The graph on the right shows the entropy; we can clearly see a second-order phase transition, as there is a discontinuity in its derivative near critical temperature.

becomes exactly zero. For $\beta J<(\beta J)_{\text {crit }}$, the only possible solution is $G_{12}(\tau)=0$. Thus, the $\mathbb{Z}_{2}$ symmetry is restored, and this is a second-order phase transition. The plot of $(\beta J)_{\text {crit }}$ vs $\alpha$ is shown in Fig. 11; it blows up as $\alpha$ approaches zero from below [78].

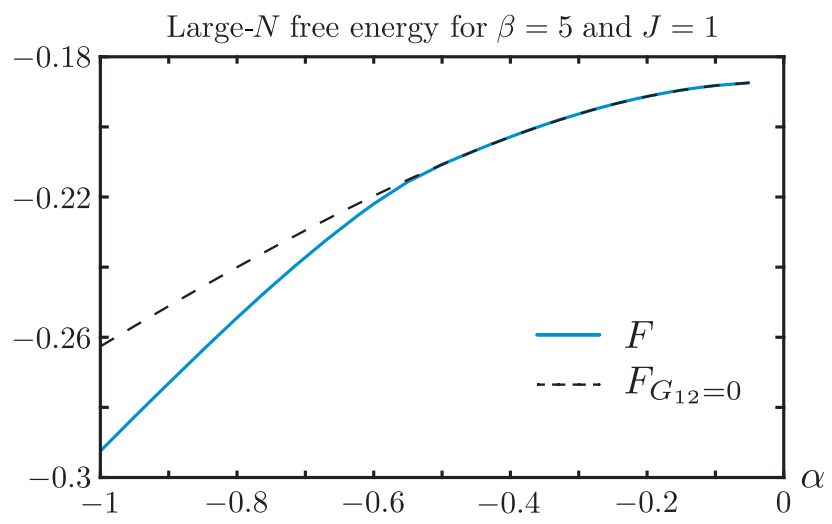

FIG. 13. Large- $N$ free energies at fixed $\beta$ and $J$. We take $\beta=5$, $J=1$, and decrease $\alpha$. We observe also a second-order phase transition.

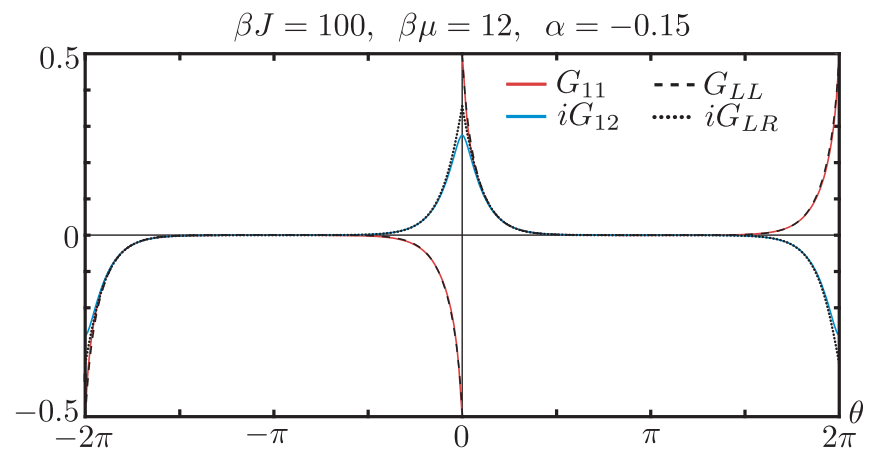

FIG. 14. Plot of solutions $G_{L L}$ and $i G_{L R}$ for the model in Ref. [44] superimposed with $G_{11}$ and $i G_{12}$. Parameters chosen so that the solutions are close for most of the range of $\theta$.
Using the solutions of the Schwinger-Dyson equations, we can numerically compute the large- $N$ free energy

$$
\begin{aligned}
-\frac{\beta F}{N_{\mathrm{SYK}}}= & \log 2+\frac{1}{2} \sum_{n=-\infty}^{+\infty} \log \left[\left(1+\frac{\Sigma_{11}\left(\omega_{n}\right)}{i \omega_{n}}\right)^{2}-\frac{\Sigma_{12}^{2}\left(\omega_{n}\right)}{\omega_{n}^{2}}\right] \\
& +\frac{3}{4} \sum_{n=-\infty}^{+\infty}\left[\Sigma_{11}\left(\omega_{n}\right) G_{11}\left(\omega_{n}\right)-\Sigma_{12}\left(\omega_{n}\right) G_{12}\left(\omega_{n}\right)\right],
\end{aligned}
$$

where the sum $\sum_{n} \log \left(-i \omega_{n}\right)$ is replaced by $\log (2)$. The energy can be computed with the formula

$\frac{E}{N_{\mathrm{SYK}}}=\frac{1}{2 \beta} \sum_{n=-\infty}^{+\infty}\left[\Sigma_{11}\left(\omega_{n}\right) G_{11}\left(\omega_{n}\right)-\Sigma_{12}\left(\omega_{n}\right) G_{12}\left(\omega_{n}\right)\right]$,

and at low temperatures it should converge to the energy of the ground state $E_{0}$ divided by $N_{\mathrm{SYK}}$.

Now one can compare the free energy in the symmetrybroken phase $F_{G_{12} \neq 0}$ with that of the symmetry-unbroken phase $F_{G_{12}=0}$. In particular, the free energy of the latter phase is simply twice that of a single SYK with a rescaling

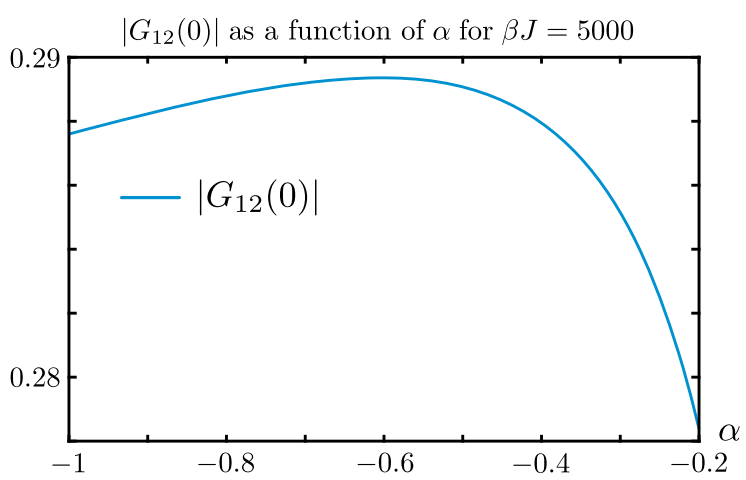

FIG. 15. The expectation value of $Q / N_{\text {SYK }}$, i.e., $\left|G_{12}(0)\right|$, as a function of $\alpha$ for $\beta J=5000$. 

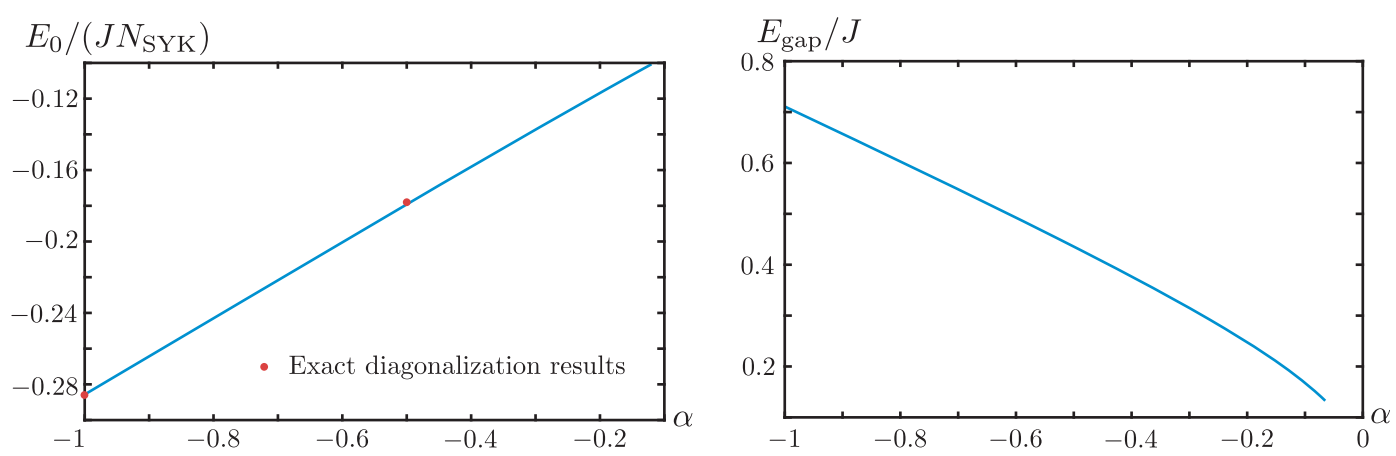

FIG. 16. Right: The value of $E_{0} /\left(J N_{\mathrm{SYK}}\right)$ as a function of $\alpha$. Both graphs are approximately linear in $\alpha$ for $\alpha$ not too small. Results from exact diagonalizations [Eq. (3.33)] are shown with dots. Left: The large- $N_{\mathrm{SYK}}$ energy gap in the spectrum computed from the exponential decay of the Green functions.
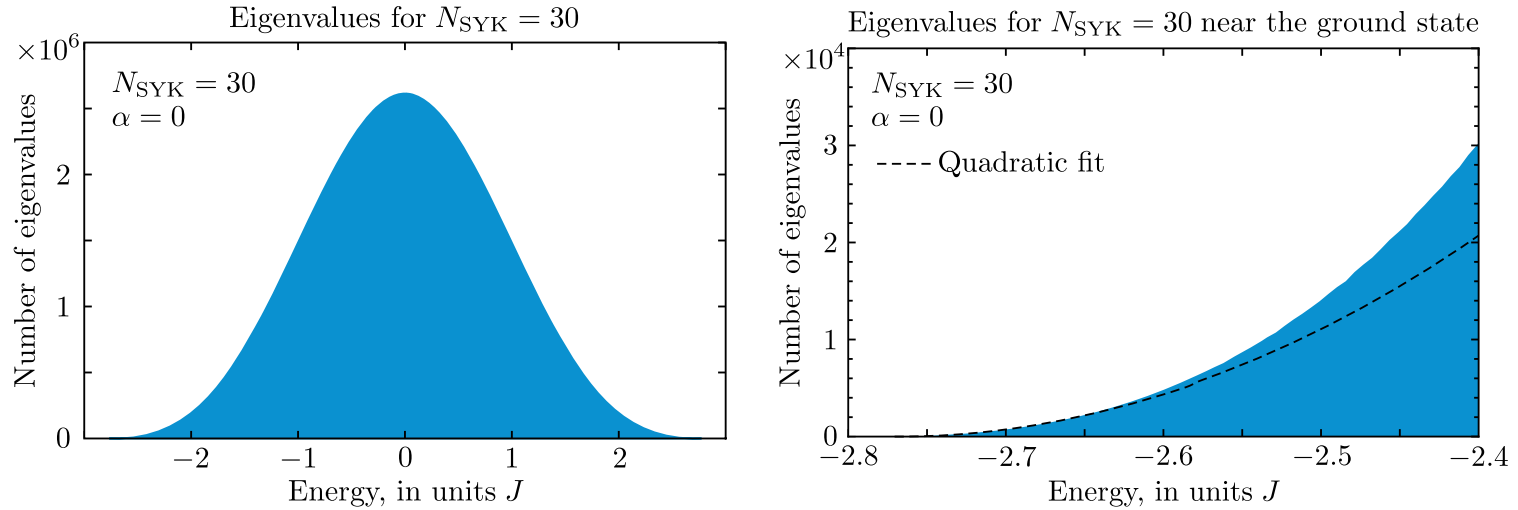

FIG. 17. Left: The energy spectrum for $\alpha=0$, i.e., for two decoupled SYK models, for a single sampling of $N_{\mathrm{SYK}}=30$. Right: The same spectrum magnified near the lower edge.

$J \rightarrow \sqrt{1+3 \alpha^{2}} J$. It follows that in the "conformal window" $0 \leq \alpha \leq 1 / 3$, the low-temperature limit of the entropy is

$$
S_{0}=2 c_{0} N, \quad c_{0}=\frac{1}{8} \log 2+\frac{K}{2 \pi} \approx 0.2324,
$$

which is twice that of the single SYK model. The fact that $S_{0}$ is independent of $\alpha$ means that the $g$ theorem [79] is obeyed to leading order in $N$, even though the theory is not exactly conformal due to the peculiarities of the $h=2$ mode. As a further check, one can consider a large- $q$ expansion $[19,80]$,

$$
\begin{aligned}
\beta F_{G_{12}=0}^{(q)}= & -\log 2-\frac{1}{q^{2}} 2 \pi \nu\left(\tan \frac{\pi \nu}{2}-\frac{\pi \nu}{4}\right) \\
& -\frac{1}{q^{3}} 2 \pi \nu\left[\pi \nu-2 \tan \pi \nu\left(1-\frac{\pi^{2} \nu^{2}}{12}\right)\right]+\ldots,
\end{aligned}
$$

where $\beta J \sqrt{\left(1+3 \alpha^{2}\right) 2^{1-q} q}=\{(\pi \nu) / \cos [(\pi \nu) / 2]\}$. The free energy of the symmetry-unbroken phase $F_{G_{12}=0}$ is seen to agree well numerically with $F_{G_{12}=0}^{(4)}$.

In Fig. 12, we plot for $\alpha=-1$ the free energy of the symmetry-broken phase (3.23) as a function of $\beta J$ and compare it with that of the unbroken phase obtained by setting $G_{12}=0$ in the SD equations (3.20) and (3.21). We also show the entropy as a function of $\beta J$. The plot shows a clear second-order phase transition at $(\beta J)_{\text {crit }} \approx 2.87$, and the derivative of the entropy is discontinuous. We also observe a second order phase transition as one fixes temperature and varies $\alpha$ as shown in Fig. 13. We will systematically study the critical exponents in future work.

We notice that at sufficiently large $\beta J$, there is a range of $\tau$ where both $i G_{12}(\tau)$ and $G_{11}(\tau)$ decay exponentially and share the same decay rate. To explain this fact, let us study the $T=0$ case and insert the complete set of states

$$
G_{11}(\tau)=\left\langle 0_{+}\left|e^{-H \tau} \chi_{1}^{1}(0) e^{H \tau}\right| n\right\rangle\left\langle n\left|\chi_{1}^{1}(0)\right| 0_{+}\right\rangle .
$$

For large $\tau$, the sum is dominated by the lowest excited state, and we find

$$
G_{11}(\tau) \rightarrow e^{-\left(E_{1}-E_{0}\right) \tau}\left\langle 0_{+}\left|\chi_{1}^{1}(0)\right| 1\right\rangle\left\langle 1\left|\chi_{1}^{1}(0)\right| 0_{+}\right\rangle .
$$

Similarly, we find that the large- $\tau$ behavior of $G_{12}$ is

$$
G_{12}(\tau) \rightarrow e^{-\left(E_{1}-E_{0}\right) \tau}\left\langle 0_{+}\left|\chi_{1}^{1}(0)\right| 1\right\rangle\left\langle 1\left|\chi_{2}^{1}(0)\right| 0_{+}\right\rangle .
$$



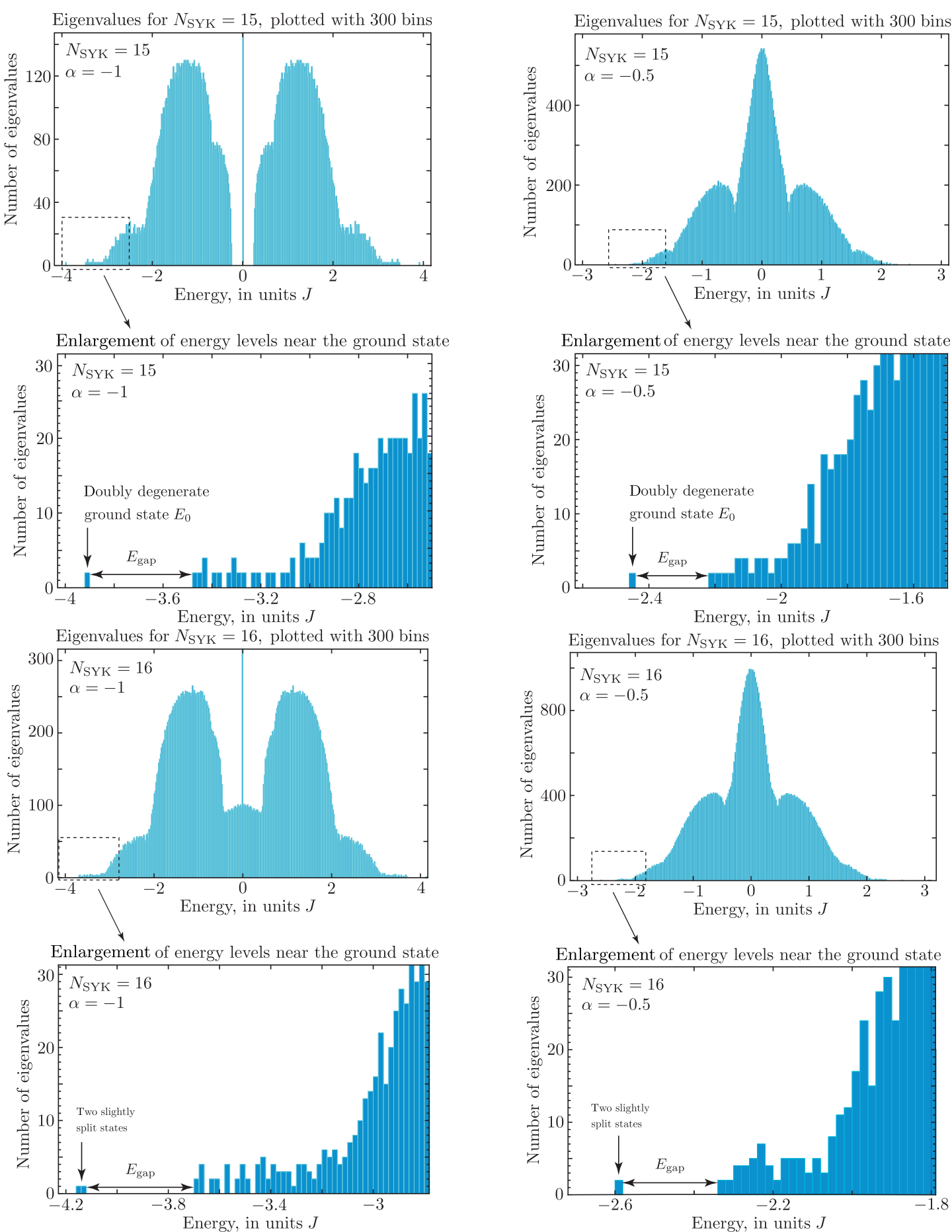

FIG. 18. The spectrum for a single realization with $N_{\mathrm{SYK}}=15,16$ and $\alpha=-1,-0.5$. For $\alpha=-1$, the spectrum exhibits a gap near $E=0$ when $N_{\mathrm{SYK}}$ is odd and a large number of states with $E=0$.

Thus, the universal decay rate among correlators signifies a mass gap in the spectrum.

In the work of Maldacena and Qi [44], the functions $G_{11}$ and $G_{12}$ were also found to be exponentially decreasing for sufficiently large $\beta J$. In Fig. 14, we exhibit superimposed plots of the low-temperature solutions to our system of equations and those from Ref. [44], with parameters chosen so that the solutions are close to one another for most of the range. We observe a difference in the behavior of $i G_{12}(\tau)$ and $i G_{L R}(\tau)$ at small $\tau$ : In our case, the function is smooth with a vanishing derivative at $\tau=0$, while in Ref. [44], its derivative is discontinuous at $\tau=0$. This is due to the fact that their Hamiltonian includes a quadratic term. 


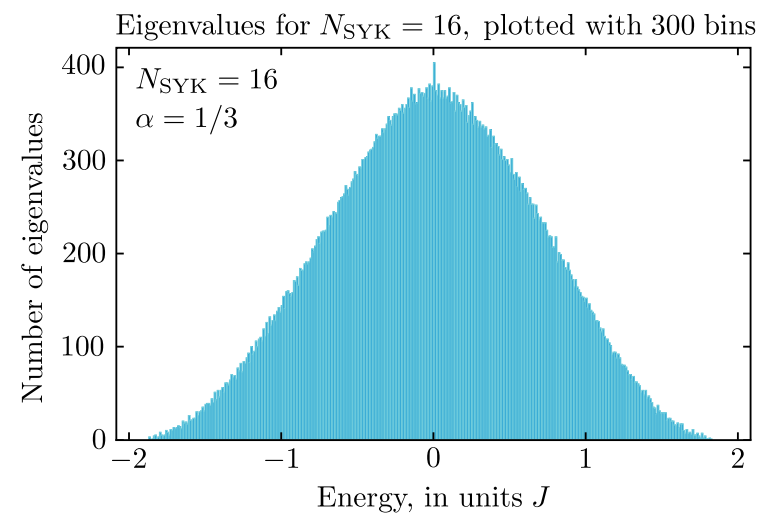

FIG. 19. The spectrum for a single realization for $N_{\mathrm{SYK}}=16$ and $\alpha=1 / 3$.

We can also study what happens at low temperatures (large $\beta J$ ) as a function of $\alpha$. In Fig. 15, we plot $i G_{12}(0)$, which is the expectation value of the order parameter $Q / N_{\mathrm{SYK}}$, for a large $\beta J$. This quantity becomes small as $\alpha$ is increased towards zero. In Fig. 16, we plot the large$N_{\text {SYK }}$ limit of the energy gap $E_{\text {gap }}$ divided by $J$ calculated from the exponential decay of the Green functions. We also plot the ground-state energy $E_{0}$ divided by $J N_{\text {SYK }}$ calculated using Eq. (3.24). The results from exact diagonalizations extrapolated to large $N_{\text {SYK }}$ [Eq. (3.33)] are shown with dots and demonstrate very good agreement. The exact diagonalizations for finite $N_{\mathrm{SYK}}$ are discussed in the next section.
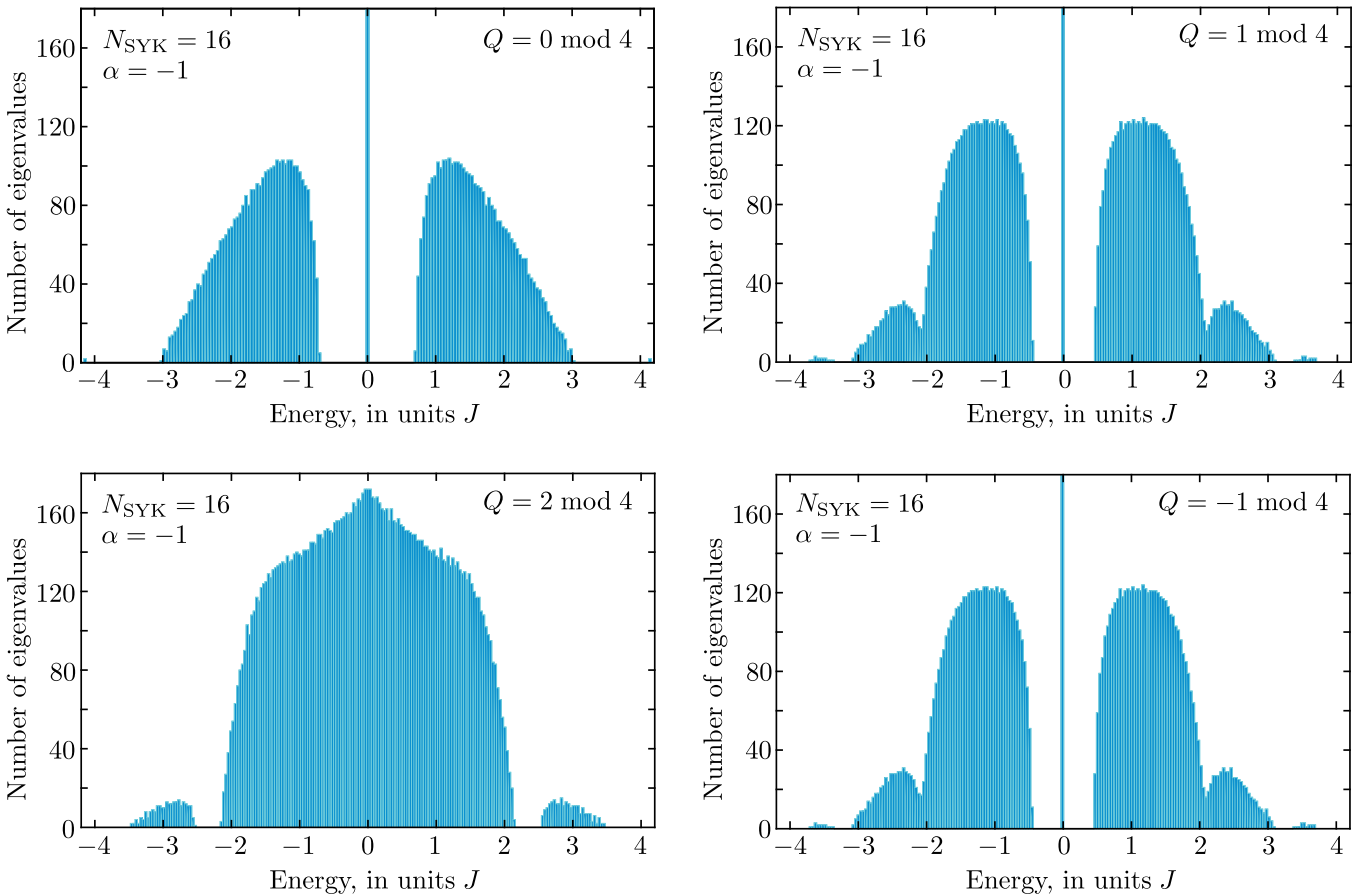

FIG. 20. The spectrum for a single realization of the coupled SYK model with $N_{\mathrm{SYK}}=16$ and $\alpha=-1$ separated into four $Z_{4}$ symmetry sectors. In each of the sectors, the spectrum is symmetric under $E \rightarrow-E$. The $Z_{4}$-invariant sector shows two nearly degenerate lowest states separated by a gap from the rest of the states. 

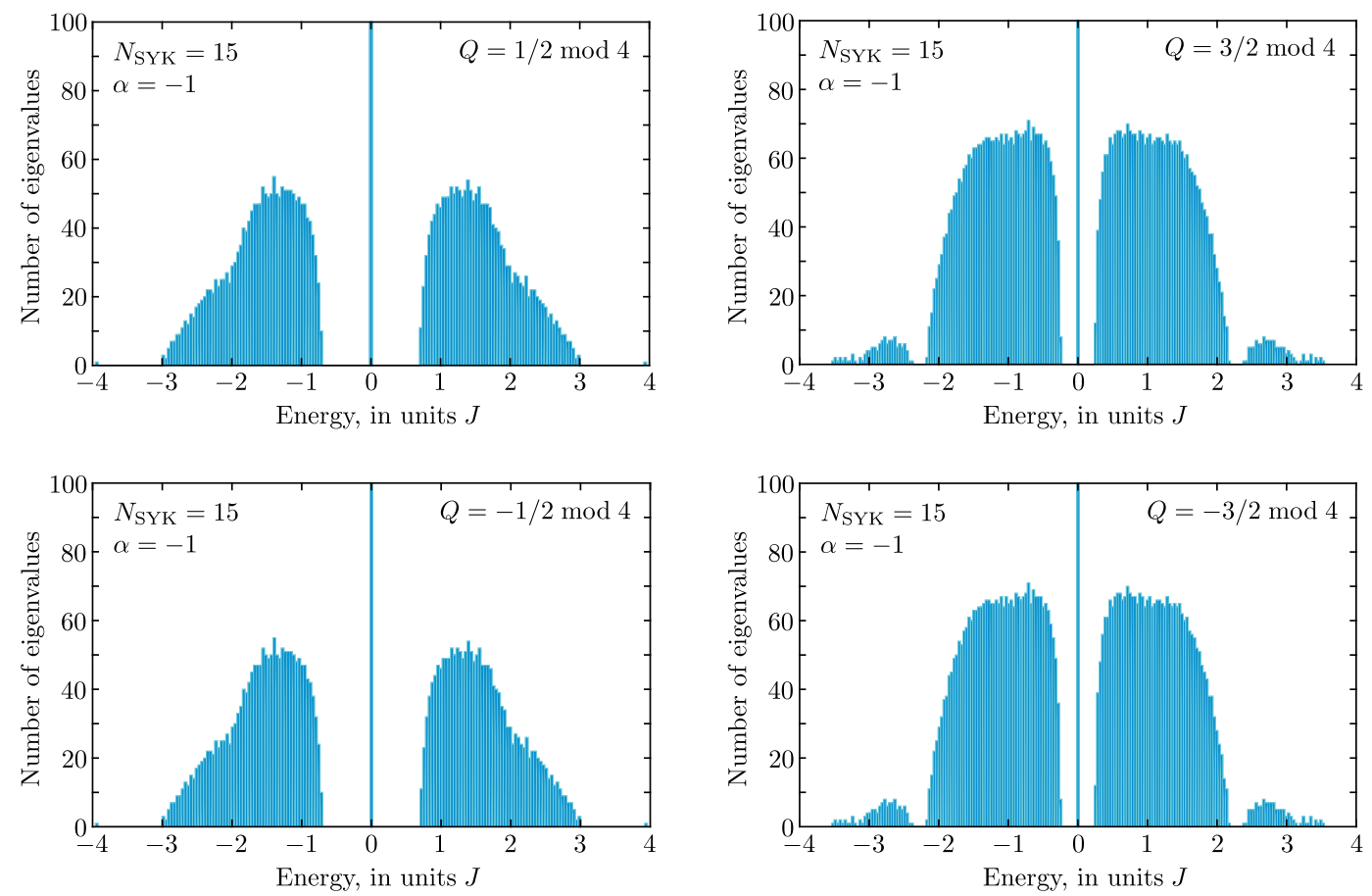

FIG. 21. The spectrum for a single realization of the coupled SYK model with $N_{\mathrm{SYK}}=15$ and $\alpha=-1$ separated into four $Z_{4}$ symmetry sectors. In each of the sectors, the spectrum is symmetric under $E \rightarrow-E$. The sectors with $Q= \pm 1 / 2 \bmod 4$ contain the ground state separated by a gap from the rest of the states.

Equation (3.30) in particular helps us determine the behavior of $\rho_{\text {double }}(E)$ near the ground state. Shifting the energy so that the ground state is at zero, we know that $\rho(E) \rightarrow A \sqrt{E}$ for small $E$. Therefore, for small $E$,

$$
\rho_{\text {double }}(E) \rightarrow A^{2} \int_{0}^{E} d e \sqrt{e(E-e)}=\frac{\pi A^{2} E^{2}}{8} .
$$

The numerical density of states shown in Fig. 17 for $N_{\mathrm{SYK}}=30$ is in good agreement with the $E^{2}$ dependence near the ground state.

Let us proceed to the spectra for nonvanishing values of $\alpha$. In Figs. 18 and 19, we plot the spectra of energy divided by $J$ for $\alpha=-1,-1 / 2,1 / 3$, and different values of $N_{\mathrm{SYK}}$. These energy distributions have interesting and unusual shapes. For the special values $\alpha=-1$ and $1 / 3$, we observe large numbers of states with $E=0$; this creates the zeroenergy peaks seen in the graphs. For $\alpha=-1$ and odd $N_{\mathrm{SYK}}$, we find that the $E=0$ peak is separated by gaps from the remaining states, but for even $N_{\mathrm{SYK}}$, it is not.

In order to clarify the peculiar shapes of the energy distributions in Fig. 18, it is useful to separate them into distinct $Z_{4}$ symmetry sectors [82] labeled by the eigenvalue of $e^{\pi i Q / 2}$, as shown in Fig. 20 for $N_{\mathrm{SYK}}=16$. The sectors where $e^{i \pi Q / 2}= \pm i$, i.e., $Q= \pm 1 \bmod 4$, have identical energy spectra which are shown on the right. They contain the symmetric bumps, which produce the "rabbit ears" pattern in the overall distribution. For $\alpha=-1$, these sectors also contain large numbers of states with $E=0$ (they are discussed in Appendix B). On the left in Fig. 20, we show the states with $e^{i \pi Q / 2}= \pm 1$. For $e^{i \pi Q / 2}=-1$, the distribution is smooth and does not contain a sharp peak at $E=0$. The $Z_{4}$-invariant sector $e^{i \pi Q / 2}=1$ contains the two nearly degenerate lowest states separated by a very clear gap from the remaining states. For $\alpha=-1$, this sector also contains a large number of $E=0$ states [83].

For $N_{\mathrm{SYK}}=15$, due to the anomaly in particle-hole symmetry, there are two degenerate ground states; see Fig. 18 [84]. In fact, each energy level is doubly degenerate. This is due to the fact that the spectra in the sectors with charges $Q=1 / 2 \bmod 4$ and with charges $Q=-1 / 2 \bmod 4$ are identical; similarly, the spectra with $Q= \pm 3 / 2 \bmod 4$ are identical. For $-1 \leq \alpha<0$, we observe a gap between the lowest energy level and the next one, as expected. The spectra for $\alpha=-1$ separated into the four sectors are shown in Fig. 21. On the other hand, for $N_{\mathrm{SYK}}=16$ there is no exact degeneracy of the ground state, but the first gap is very small, indicating a tendency towards spontaneous symmetry breaking at large $N_{\mathrm{SYK}}$. We show the $N_{\mathrm{SYK}}=16$ spectra for $\alpha=-1$ and $\alpha=-0.5$ in Fig. 18. In both cases, for a typical sampling of the coupling constants $J_{i j k l}$, we observe two closely spaced states followed by a visible gap. For large $N_{\mathrm{SYK}}$, the energy gap between the two lowest states is expected to decrease exponentially: 

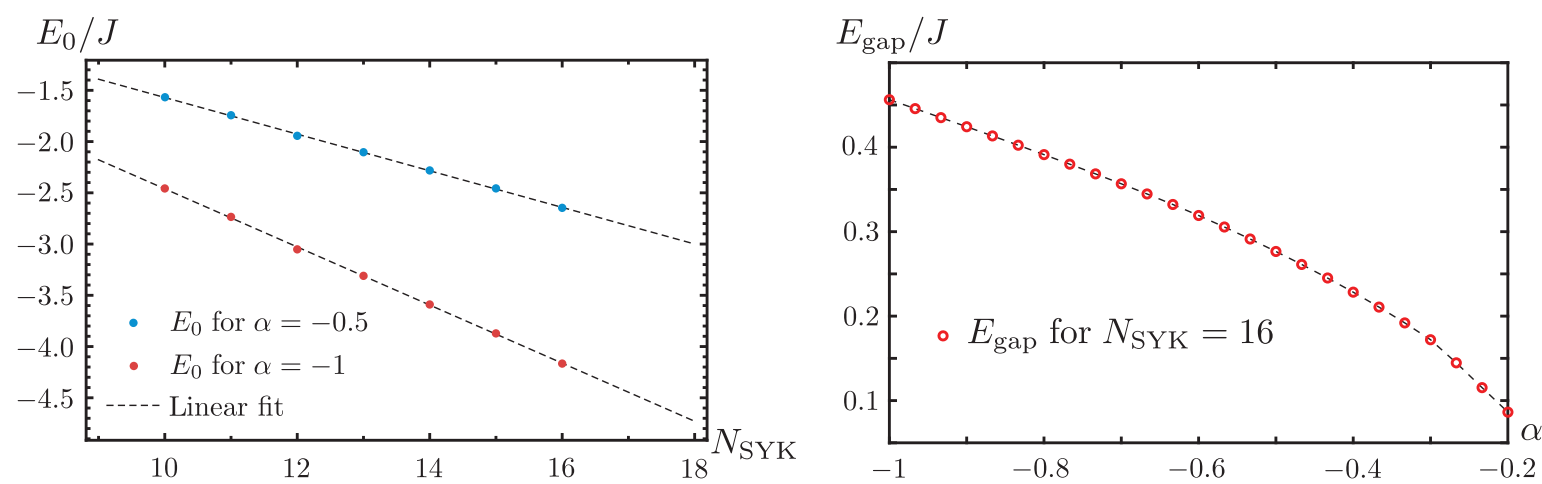

FIG. 22. Left: The ground-state energy for $\alpha=-0.5,-1$ and $N_{\mathrm{SYK}}=10,11, \ldots, 16$ (the number of samples are 250000,120000 , $50000,5000,5000,2000,500)$. The linear fit is shown by dashed lines. Right: The energy gap between the second and third states as a function of $\alpha$ for a single realization of random couplings at $N_{\mathrm{SYK}}=16$.

$$
-\log \frac{E_{1}-E_{0}}{J} \sim N_{\mathrm{SYK}}
$$

For $\alpha \geq 0$, the low-lying spectrum is different; we observe many closely spaced low-lying states without large gaps, similar to the standard SYK spectrum.

In Fig. 22, we plot the ground-state energy for $\alpha=-1$ and $\alpha=-0.5$ with $N_{\mathrm{SYK}}=10, \ldots, 16$. The plots where $J$ is set to 1 are approximately linear, and the fits give

$$
\begin{aligned}
E_{0}^{\alpha=-1} & =-0.283 N_{\mathrm{SYK}}+0.373, \\
E_{0}^{\alpha=-0.5} & =-0.179 N_{\mathrm{SYK}}+0.217 .
\end{aligned}
$$

The limiting values $E_{0}^{\alpha=-1} / N_{\text {SYK }}=-0.283$ and $E_{0}^{\alpha=-0.5} /$ $N_{\text {SYK }}=-0.179$ are in good agreement with the result found from the Schwinger-Dyson equations; see Fig. 16. In Fig. 22, we also exhibit the energy gap between the second and third states as a function of $\alpha$. As $\alpha$ is increased from -1 to 0 , the gap decreases as expected.

Exact diagonalizations also provide support for the statement that the fermion number $Q$ acquires a vacuum expectation value for $-1 \leq \alpha<0$. For $N_{\mathrm{SYK}}$ not divisible by 4 , there are two ground states $\left|0_{ \pm}\right\rangle$which map into each

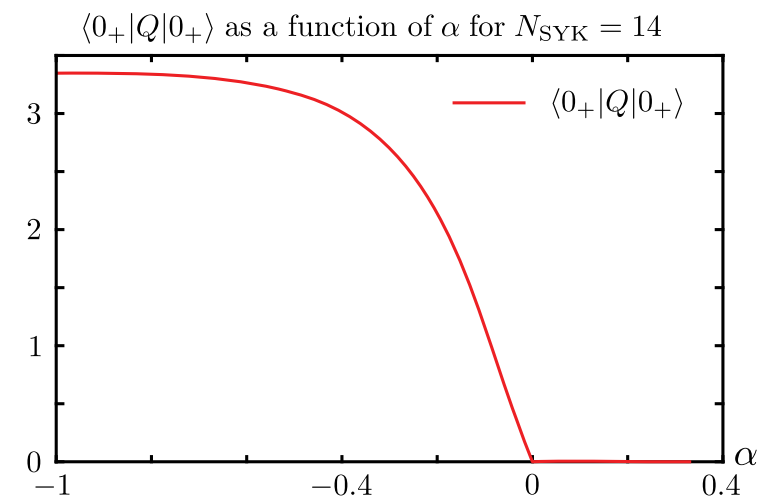

FIG. 23. The expectation value $\left\langle 0_{+}|Q| 0_{+}\right\rangle$as a function of $\alpha$ for a single realization of random couplings at $N_{\mathrm{SYK}}=14$. other under the symmetry generator $\mathcal{P}$. This can be viewed as anomalous breaking of the time-reversal $\mathbb{Z}_{2}$ symmetry (3.11) which occurs for a finite number of degrees of freedom [37-39,41,42]. In Fig. 23, the vacuum expectation value as a function of $\alpha$ is plotted for $N_{\mathrm{SYK}}=14$. This is the finite $N_{\mathrm{SYK}}$ analogue of Fig. 15 , where the large- $N_{\mathrm{SYK}}$ limit of the condensate is plotted. We also note the qualitative similarity of Fig. 23 and that of the imaginary part of the scaling dimension of $Q$ in Fig. 8.

\section{ACKNOWLEDGMENTS}

Some of the results presented here are from J. K.'s Princeton University senior thesis [65]. I. R. K. is grateful to the Kavli Institute for Theoretical Physics at University of California, Santa Barbara and the organizers of the program "Chaos and Order: From Strongly Correlated Systems to Black Holes" for the hospitality and stimulating atmosphere during some of his work on this paper. His research at Kavli Institute for Theoretical Physics is supported in part by the National Science Foundation under Grant No. NSF PH-1748958. I. R. K. is also grateful to the participants of the program and especially D. Gross, C.-M. Jian, A. Kitaev, J. Maldacena, D. Stanford, J. Verbaarschot, E. Witten, and C. Xu for very useful discussions. G. T. would like to thank D. Jafferis for useful discussions. The work of I. R. K. and W.Z. is supported in part by the U.S. National Science Foundation under Grant No. PHY-1620059. The work of G. T. is supported by the MURI Grant No. W911NF-14-10003 from ARO and by Department of Energy Grants No. DE-SC0007870 and No. DE-SC0019030.

\section{APPENDIX A: MORE ON THE DISCRETE SYMMETRIES}

The model (1.1) has the antiunitary particle-hole $\mathbb{Z}_{2}$ symmetry generated by Eq. (3.11). The operator $K$ is defined to take $z \rightarrow \bar{z}, z \in \mathbb{C}$ but acts as the identity on $\psi$ or $\bar{\psi}$. It may be identified as a kind of time-reversal generator which satisfies $K^{2}=1$ [37-39]. It acts by 


$$
K i K=-i, \quad K \chi_{1}^{i} K=\chi_{1}^{i}, \quad K \chi_{2}^{i} K=-\chi_{2}^{i},
$$

and therefore satisfies

$$
[K, H]=[K, Q]=0 .
$$

Note that although $K$ can be anomalous, $K$ is unbroken as it does not change the sign of $Q$. Another unbroken symmetry is the $\pi / 2$ rotation between $\chi_{1}^{i}$ and $\chi_{2}^{i}$,

$$
R=(-1)^{N_{\mathrm{SYK}} / 4} 2^{-N_{\mathrm{SYK}} / 2} \prod_{i}\left(1-2 \chi_{1}^{i} \chi_{2}^{i}\right) .
$$

It satisfies

$R R^{\dagger}=1, \quad R \chi_{1}^{i} R^{\dagger}=\chi_{2}^{i}, \quad R \chi_{2}^{i} R^{\dagger}=-\chi_{1}^{i}, \quad R^{4}=1$.

Note $R^{2}=(-1)^{F}$. There are also various reflection $\mathbb{Z}_{2}$ symmetries that are spontaneously broken by the VEV of $Q$. In particular, we have the reflection symmetry

$$
P= \begin{cases}(-1)^{N_{\mathrm{SYK}}\left(N_{\mathrm{SYK}}-1\right) / 4} 2^{N_{\mathrm{SYK}} / 2} \prod_{i=1}^{N_{\mathrm{SYK}}} \chi_{1}^{i} & \text { if } N_{\mathrm{SYK}}=2 k, k \in \mathbb{Z}, \\ (-1)^{N_{\mathrm{SYK}}\left(N_{\mathrm{SYK}}-1\right) / 4} 2^{N_{\mathrm{SYK}} / 2} \prod_{i=1}^{N_{\mathrm{SYK}}} \chi_{2}^{i} & \text { if } N_{\mathrm{SYK}}=2 k+1, k \in \mathbb{Z},\end{cases}
$$

such that

$$
P P^{\dagger}=1, \quad P \chi_{1}^{i} P^{\dagger}=-\chi_{1}^{i}, \quad P \chi_{2}^{i} P^{\dagger}=\chi_{2}^{i}, \quad P^{2}=1 .
$$

In fact, $R, P$, and $K$ are enough to generate all discrete symmetries of the model (1.1). In particular, all the unitary discrete symmetries form $D_{4}$, the dihedral group of order 8. To see this, it is enough to check that the group presentation $R^{4}=P^{2}=(R P)^{2}=1$. The remaining reflections can be identified with $R P, R^{2} P$, and $R^{3} P$. For a given unitary symmetry, we can compose it with $K$ to obtain an antiunitary one.

In our case, when $N_{\text {SYK }} \rightarrow \infty$, although multiple $\mathbb{Z}_{2}$ symmetries are spontaneously broken, we expect only a twofold ground-state degeneracy. In fact, any two broken symmetries that can be related by an unbroken symmetry do not produce any extra ground-state degeneracy. To see this, consider for example the reflection symmetry $R P$. Since $R$ is unbroken, we may assume $R|0\rangle=|0\rangle$ without loss of generality. Then, $R P|0\rangle=R P R|0\rangle=P|0\rangle$.

At finite $N_{\text {SYK }}$, however, certain discrete symmetry can be anomalous and is responsible for an exact twofold degeneracy for certain $N_{\mathrm{SYK}}$. For example, the particle-hole symmetry $\mathcal{P} \sim K P$ acts on the fermions as

$$
\begin{aligned}
\mathcal{P} \psi^{j} \mathcal{P} & =\eta \bar{\psi}^{j}, \quad \mathcal{P} \bar{\psi}^{j} \mathcal{P}=\eta \psi^{j}, \\
\eta & =(-1)^{\left(N_{\mathrm{SYK}}+2\right)\left(N_{\mathrm{SYK}}-1\right) / 2} .
\end{aligned}
$$

The fermion number operator (3.7) is odd under this symmetry:

$$
\mathcal{P} Q \mathcal{P}=-\mathcal{P}^{2} Q .
$$

When $N_{\text {SYK }}$ is not divisible by 4 , there are two degenerate ground states $\left|0_{ \pm}\right\rangle$, and the symmetry generator $\mathcal{P}$ maps them into each other [37-42]:

$$
\begin{aligned}
& \mathcal{P}\left|0_{+}\right\rangle=(-1)^{N_{\mathrm{SYK}}\left(N_{\mathrm{SYK}}-1\right) / 4}\left|0_{-}\right\rangle, \\
& \mathcal{P}\left|0_{-}\right\rangle=(-1)^{N_{\mathrm{SYK}}\left(N_{\mathrm{SYK}}-1\right) / 4}\left|0_{+}\right\rangle .
\end{aligned}
$$

In this case, we can say that the particle-hole symmetry is anomalous.

\section{APPENDIX B: ZERO-ENERGY STATES IN THE BIPARTITE MODEL}

The bipartite model, which is the $\alpha=-1$ case of the two-flavor tensor or SYK model, has some additional symmetries which make it special. In general, the spectrum of the two-flavor SYK is not symmetric under $E \rightarrow-E$ for a given random coupling $J_{i j k l}$. However, for $\alpha=-1$, the spectrum is exactly symmetric for any choice $J_{i j k l}$ due to the duality symmetry (2.11). This symmetry acts by

$$
\psi^{j} \rightarrow \frac{1+i}{\sqrt{2}} \bar{\psi}^{j}, \quad \bar{\psi}^{j} \rightarrow \frac{1-i}{\sqrt{2}} \psi^{j},
$$

and for $\alpha=-1$ this reverses the sign of the Hamiltonian of bipartite model $H_{\alpha=-1}$, which is given in Eq. (3.6).

Furthermore, the model with $\alpha=-1$ has a large number of zero-energy states. For the SYK model, the sharp peak at $E=0$ may be seen in Fig. 18. For a generic choice of $J_{i j k l}$ where they are all nonvanishing, the number of $E=0$ states does not depend on their values. In fact, it is not hard to calculate this number separately for each $Z_{4}$ symmetry sector. The separate sectors may be labeled by $Q=\tilde{Q} \bmod 4$, where $\tilde{Q}=0, \pm 1,2$ when $N$ is even, and $\tilde{Q}= \pm 1 / 2, \pm 3 / 2$ when $N$ is odd [85]. The general formula for the number of $E=0$ states in sector $\tilde{Q}$ is

$$
\mathcal{N}_{\tilde{Q}}=\sum_{m=-[(N+2 \tilde{Q}) / 8]}^{[(N-2 \tilde{Q}) / 8]}(-1)^{m}\left(\begin{array}{c}
N \\
\frac{N}{2}+\tilde{Q}+4 m
\end{array}\right) .
$$

This formula is applicable to "generic" bipartite Hamiltonians (3.6), where all $J_{i j k l}$ are nonvanishing; in 


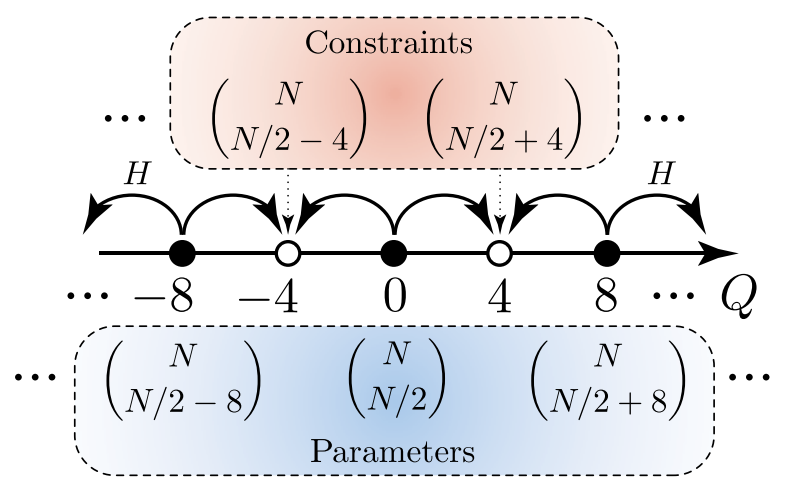

FIG. 24. This picture represents the counting of zero-energy states in the $\tilde{Q}=0$ sector. They are superpositions of states with charges $Q=0 \bmod 8$.

such cases, it does not depend on the specific choice of couplings. However, if some couplings $J_{i j k l}$ vanish, then the number of $E=0$ states may be higher than Eq. (B2). For example, in the $O\left(N_{1}\right) \times O\left(N_{2}\right) \times O\left(N_{3}\right)$ tensor bipartite models, where many quartic couplings vanish [17], the number of $E=0$ states is greater than that given by Eq. (B2) with $N=N_{1} N_{2} N_{3}$.

To explain the origin of the formula (B2), let us consider for example the $\tilde{Q}=0$ sector of a model with even $N_{\mathrm{SYK}}$. In this sector, the $E=0$ states may be obtained from superpositions of states with $Q=0 \bmod 8$ [86]. The dimension of Hilbert space in this sector is

$$
d_{0 \bmod 8}=\sum_{m}\left(\begin{array}{c}
N \\
\frac{N}{2}+8 m
\end{array}\right)
$$

When the Hamiltonian of the bipartite model acts on such a state, it maps it to a superposition of states with $Q=4 \mathrm{mod}$ 8 (see Fig. 24). The total number of such states is $\sum_{m}\left(\begin{array}{c}N \\ \frac{N}{2}+4+8 m\end{array}\right)$, and this is the number of constraints from the requirement that $H_{\alpha=-1}$ annihilates the zero-energy states. Subtracting this number of constraints from $d_{0 \bmod 8}$, we arrive at Eq. (B2) for the case $\tilde{Q}=0$. Analogous reasoning provides a derivation of Eq. (B2) for other values of $\tilde{Q}$. We check numerically that all the $E=0$ wave functions are mixtures of only the states with $Q=\tilde{Q} \bmod 8$ and that their numbers for any random sampling of $J_{i j k l}$ are given by Eq. (B2).

For example, for $N=16$ the number of states in the $\tilde{Q}=0$ sector is

$$
\mathcal{N}_{0}=\left(\begin{array}{c}
16 \\
8
\end{array}\right)+2\left(\begin{array}{c}
16 \\
16
\end{array}\right)-2\left(\begin{array}{c}
16 \\
4
\end{array}\right)=9232
$$

The number of states in the $\tilde{Q}= \pm 1$ sectors is

$$
\mathcal{N}_{1}=\mathcal{N}_{-1}=\left(\begin{array}{c}
16 \\
7
\end{array}\right)+\left(\begin{array}{c}
16 \\
15
\end{array}\right)-\left(\begin{array}{c}
61 \\
3
\end{array}\right)-\left(\begin{array}{l}
16 \\
11
\end{array}\right)=6528
$$

The number of states in the $\tilde{Q}=2$ sector vanishes for any even $N$.

[1] S. Sachdev and J. Ye, Gapless Spin Fluid Ground State in a Random, Quantum Heisenberg Magnet, Phys. Rev. Lett. 70, 3339 (1993).

[2] A. Kitaev, A Simple Model of Quantum Holography, in Proceedings of KITP, April 7, 2015 and May 27, 2015, http://online.kitp.ucsb.edu/online/entangled15/kitaev/, http://online.kitp.ucsb.edu/online/entangled15/kitaev2/.

[3] S. Sachdev, Bekenstein-Hawking Entropy and Strange Metals, Phys. Rev. X 5, 041025 (2015).

[4] A. Kitaev and S. J. Suh, The Soft Mode in the Sachdev-YeKitaev Model and Its Gravity Dual, J. High Energy Phys. 05 (2018) 183.

[5] E. Witten, An SYK-Like Model without Disorder, arXiv: 1610.09758.

[6] I. R. Klebanov and G. Tarnopolsky, Uncolored Random Tensors, Melon Diagrams, and the Sachdev-Ye-Kitaev Models, Phys. Rev. D 95, 046004 (2017).

[7] R. Gurau, Colored Group Field Theory, Commun. Math. Phys. 304, 69 (2011).

[8] R. Gurau and V. Rivasseau, The 1/N Expansion of Colored Tensor Models in Arbitrary Dimension, Europhys. Lett. 95, 50004 (2011).

[9] R. Gurau, The Complete 1/N Expansion of Colored Tensor Models in Arbitrary Dimension, Ann. Inst. Henri Poincaré 13, 399 (2012).

[10] V. Bonzom, R. Gurau, A. Riello, and V. Rivasseau, Critical Behavior of Colored Tensor Models in the Large N Limit, Nucl. Phys. B853, 174 (2011).

[11] A. Tanasa, Multi-Orientable Group Field Theory, J. Phys. A 45, 165401 (2012).

[12] V. Bonzom, R. Gurau, and V. Rivasseau, Random Tensor Models in the Large $N$ Limit: Uncoloring the Colored Tensor Models, Phys. Rev. D 85, 084037 (2012).

[13] S. Carrozza and A. Tanasa, $O(N)$ Random Tensor Models, Lett. Math. Phys. 106, 1531 (2016).

[14] R. Gurau and J.P. Ryan, Colored Tensor Models-A Review, SIGMA 8, 020 (2012).

[15] A. Tanasa, The Multi-Orientable Random Tensor Model, A Review, SIGMA 12, 056 (2016).

[16] N. Delporte and V. Rivasseau, The Tensor Track V: Holographic Tensors, arXiv:1804.11101.

[17] I. R. Klebanov, F. Popov, and G. Tarnopolsky, TASI Lectures on Large N Tensor Models, Proc. Sci., TASI2017 (2018) 004 [arXiv:1808.09434].

[18] J. Polchinski and V. Rosenhaus, The Spectrum in the Sachdev-Ye-Kitaev Model, J. High Energy Phys. 04 (2016) 001.

[19] J. Maldacena and D. Stanford, Comments on the SachdevYe-Kitaev Model, Phys. Rev. D 94, 106002 (2016). 
[20] D. J. Gross and V. Rosenhaus, A Generalization of SachdevYe-Kitaev, J. High Energy Phys. 02 (2017) 093.

[21] A. Jevicki, K. Suzuki, and J. Yoon, Bi-Local Holography in the SYK Model, J. High Energy Phys. 07 (2016) 007.

[22] A. Almheiri and J. Polchinski, Models of $\mathrm{AdS}_{2}$ Backreaction and Holography, J. High Energy Phys. 11 (2015) 014.

[23] K. Jensen, Chaos in $\mathrm{AdS}_{2}$ Holography, Phys. Rev. Lett. 117, 111601 (2016).

[24] J. Maldacena, D. Stanford, and Z. Yang, Conformal Symmetry and Its Breaking in Two Dimensional Nearly Anti-deSitter Space, Prog. Theor. Exp. Phys. 2016, 12C104 (2016).

[25] J. Engelsoy, T. G. Mertens, and H. Verlinde, An Investigation of $\mathrm{AdS}_{2}$ Backreaction and Holography, J. High Energy Phys. 07 (2016) 139.

[26] Y. Gu, X.-L. Qi, and D. Stanford, Local Criticality, Diffusion and Chaos in Generalized Sachdev-Ye-Kitaev Models, J. High Energy Phys. 05 (2017) 125.

[27] A. R. Kolovsky and D. L. Shepelyansky, Dynamical Thermalization in Isolated Quantum Dots and Black Holes, Europhys. Lett. 117, 10003 (2017).

[28] Z. Bi, C.-M. Jian, Y.-Z. You, K. A. Pawlak, and C. Xu, Instability of the Non-Fermi Liquid State of the Sachdev-YeKitaev Model, Phys. Rev. B 95, 205105 (2017).

[29] A. Haldar and V. B. Shenoy, Strange Half-Metals and Mott Insulators in Sachdev-Ye-Kitaev Models, Phys. Rev. B 98, 165135 (2018).

[30] X.-Y. Song, C.-M. Jian, and L. Balents, A Strongly Correlated Metal Built from Sachdev-Ye-Kitaev Models, Phys. Rev. Lett. 119, 216601 (2017).

[31] S.-K. Jian, Z.-Y. Xian, and H. Yao, Quantum Criticality and Duality in the Sachdev-Ye-Kitaev/AdS 2 Chain, Phys. Rev. B 97, 205141 (2018).

[32] A. A. Patel and S. Sachdev, Critical Strange Metal from Fluctuating Gauge Fields in a Solvable Random Model, Phys. Rev. B 98, 125134 (2018).

[33] S. Banerjee and E. Altman, Solvable Model for a Dynamical Quantum Phase Transition from Fast to Slow Scrambling, Phys. Rev. B 95, 134302 (2017).

[34] T. Azeyanagi, F. Ferrari, and F. I. Schaposnik Massolo, Phase Diagram of Planar Matrix Quantum Mechanics, Tensor, and Sachdev-Ye-Kitaev Models, Phys. Rev. Lett. 120, 061602 (2018).

[35] W. Fu, Y. Gu, S. Sachdev, and G. Tarnopolsky, $\mathbb{Z}_{2}$ Fractionalized Phases of a Solvable, Disordered, $t-J$ Model, Phys. Rev. B 98, 075150 (2018).

[36] This model seems similar to a coupled SYK model introduced in Ref. [26], but there each of the three terms in the Hamiltonian would have an independent random coupling. As a result, the Schwinger-Dyson equations are different from those for theory (1.1). The complex scaling dimension and symmetry breaking, which we describe in this paper, do not appear in the model of Ref. [26].

[37] A. Kitaev, Periodic Table for Topological Insulators and Superconductors, AIP Conf. Proc. 1134, 22 (2009).

[38] L. Fidkowski and A. Kitaev, The Effects of Interactions on the Topological Classification of Free Fermion Systems, Phys. Rev. B 81, 134509 (2010).

[39] E. Witten, Fermion Path Integrals and Topological Phases, Rev. Mod. Phys. 88, 035001 (2016).
[40] Y.-Z. You, A. W. W. Ludwig, and C. Xu, Sachdev-Ye-Kitaev Model and Thermalization on the Boundary of Many-Body Localized Fermionic Symmetry-Protected Topological States, Phys. Rev. B 95, 115150 (2017).

[41] W. Fu and S. Sachdev, Numerical Study of Fermion and Boson Models with Infinite-Range Random Interactions, Phys. Rev. B 94, 035135 (2016).

[42] J. S. Cotler, G. Gur-Ari, M. Hanada, J. Polchinski, P. Saad, S. H. Shenker, D. Stanford, A. Streicher, and M. Tezuka, Black Holes and Random Matrices, J. High Energy Phys. 05 (2017) 118.

[43] When $N_{\text {SYK }}$ is finite and not divisible by 4 so that the total number of Majorana fermions is not divisible by 8 , the particle-hole symmetry is broken by a discrete anomaly [37-42].

[44] J. Maldacena and X.-L. Qi, Eternal Traversable Wormhole, arXiv: 1804.00491.

[45] A. M. García-García, T. Nosaka, D. Rosa, and J. J. M. Verbaarschot, Quantum Chaos Transition in a Two-Site SYK Model Dual to an Eternal Traversable Wormhole, arXiv:1901.06031.

[46] P. Gao, D. L. Jafferis, and A. Wall, Traversable Wormholes via a Double Trace Deformation, J. High Energy Phys. 12 (2017) 151.

[47] J. Maldacena, D. Stanford, and Z. Yang, Diving into Traversable Wormholes, Fortschr. Phys. 65, 1700034 (2017).

[48] A. Dymarsky, I. R. Klebanov, and R. Roiban, Perturbative Search for Fixed Lines in Large N Gauge Theories, J. High Energy Phys. 08 (2005) 011.

[49] E. Pomoni and L. Rastelli, Large N Field Theory and AdS Tachyons, J. High Energy Phys. 04 (2009) 020.

[50] D. Grabner, N. Gromov, V. Kazakov, and G. Korchemsky, Strongly $\gamma$-Deformed $\mathcal{N}=4$ Supersymmetric Yang-Mills Theory as an Integrable Conformal Field Theory, Phys. Rev. Lett. 120, 111601 (2018).

[51] S. Prakash and R. Sinha, A Complex Fermionic Tensor Model in d Dimensions, J. High Energy Phys. 02 (2018) 086.

[52] V. Gorbenko, S. Rychkov, and B. Zan, Walking, Weak FirstOrder Transitions, and Complex CFTs, J. High Energy Phys. 10 (2018) 108.

[53] J. M. Maldacena, The Large N Limit of Superconformal Field Theories and Supergravity, Adv. Theor. Math. Phys. 2, 231 (1998).

[54] S. S. Gubser, I. R. Klebanov, and A. M. Polyakov, Gauge Theory Correlators from Noncritical String Theory, Phys. Lett. B 428, 105 (1998).

[55] E. Witten, Anti-de Sitter Space and Holography, Adv. Theor. Math. Phys. 2, 253 (1998).

[56] P. Breitenlohner and D. Z. Freedman, Stability in Gauged Extended Supergravity, Ann. Phys. (N.Y.) 144, 249 (1982).

[57] D. B. Kaplan, J.-W. Lee, D. T. Son, and M. A. Stephanov, Conformality Lost, Phys. Rev. D 80, 125005 (2009).

[58] S. Giombi, I. R. Klebanov, and G. Tarnopolsky, Conformal $\mathrm{QED}_{d}$, F-Theorem and the $\epsilon$ Expansion, J. Phys. A 49, 135403 (2016).

[59] S. Giombi, I. R. Klebanov, and G. Tarnopolsky, Bosonic Tensor Models at Large $N$ and Small $\epsilon$, Phys. Rev. D 96, 106014 (2017). 
[60] S. Giombi, I. R. Klebanov, F. Popov, S. Prakash, and G. Tarnopolsky, Prismatic Large $N$ Models for Bosonic Tensors, Phys. Rev. D 98, 105005 (2018).

[61] R. Gurau, The Complete 1/N Expansion of a SYK-like Tensor Model, arXiv:1611.04032.

[62] In Refs. [28,31], quartic interactions were added to SYK models, which have a "double-trace" structure and contain an additional random tensor $C_{i j}$. These interactions do not have a tensor counterpart because $\psi_{1}^{a b c} \psi_{1}^{a b c}$ is a $c$ number.

[63] On the other hand, in the approach of Ref. [44], where the quadratic term $\mu Q$ was added to couple the two SYK models, the gap (and therefore, the wormhole) appeared for either sign of $\mu$.

[64] This section is based in part on Kim's Princeton University senior thesis [65].

[65] J. Kim, Large N Tensor and SYK Models, Senior thesis, Princeton University, 2018 [arXiv:1811.04330].

[66] The Hamiltonian also has discrete symmetries which do not involve $K$, which combine into the dihedral group $D_{4}$. This is discussed in detail for the coupled SYK counterpart in Sec. III and in the Appendix.

[67] Using antisymmetry of the tensor $J_{I J K L}$, one can operate with Majorana fermions as commuting variables but keeping order of $I, J, K, L$ indices fixed.

[68] For the original Hamiltonian (2.14), this transformation rescales the energy levels. Therefore, our results for dimensionful quantities, like energy levels and Green functions, do not respect the duality under Eq. (2.13).

[69] In the coupled SYK model (1.1), the same expressions for bilinear operators are applicable after replacement of $\psi_{A}^{I}$ by $\chi_{A}^{i}$, with $A=1,2$.

[70] To take the integrals, one should use star-triangle identities twice [4].

[71] I. R. Klebanov and E. Witten, AdS/CFT Correspondence and Symmetry Breaking, Nucl. Phys. B556, 89 (1999).
[72] E. Witten, Multitrace Operators, Boundary Conditions, and AdS/CFT Correspondence, arXiv:hep-th/0112258.

[73] S. S. Gubser and I. R. Klebanov, A Universal Result on Central Charges in the Presence of Double Trace Deformations, Nucl. Phys. B656, 23 (2003).

[74] Because of a technicality, we expect only a twofold degeneracy although multiple $\mathbb{Z}_{2}$ 's are been broken. We comment on this issue below.

[75] This model is similar to the complex SYK model [3], but in Eq. (3.8) the coupling $J_{i j k l}$ is taken to be fully antisymmetric.

[76] These equations are also valid in the two-flavor tensor model (1.2), where $G_{a b}(\tau)=\left(1 / N^{3}\right)\left\langle T \psi_{a}^{I}(\tau) \psi_{b}^{I}(0)\right\rangle$.

[77] In this case, we find it more convenient to use a slow decay rate on the weight $x$.

[78] We note that this function does not have a vanishing derivative at the self-dual value of $\alpha=-1$. If we plot the critical value of $\beta \tilde{J}=\beta J \sqrt{|\tilde{\alpha}|}$, this derivative will vanish, but the plot would not be monotonic.

[79] I. Affleck and A. W. W. Ludwig, Universal Noninteger "Ground State Degeneracy" in Critical Quantum Systems, Phys. Rev. Lett. 67, 161 (1991).

[80] G. Tarnopolsky, Large $q$ Expansion in the Sachdev-YeKitaev Model, Phys. Rev. D 99, 026010 (2019).

[81] We thank D. Stanford for a useful discussion about this.

[82] We are very grateful to J. Verbaarschot for raising a question about separation of the spectra into sectors.

[83] If we gauge the $Z_{4}$ symmetry, then only the sector with $e^{i \pi Q / 2}=1$ will remain in the spectrum.

[84] If we instead adopt the Maldacena-Qi Hamiltonian with a quadratic coupling which breaks the particle-hole symmetry explicitly, there is no such double degeneracy.

[85] In this Appendix, $N$ denotes $N_{\text {SYK }}$.

[86] This may be interpreted as the fact that in the zero-energy sector there is symmetry enhancement from $Z_{4}$ to $Z_{8}$. 